\title{
MiCHEL OLIVIER
}

\section{Corps sextiques primitifs (IV)}

Séminaire de Théorie des Nombres de Bordeaux, tome 3, $\mathrm{n}^{\circ} 2$ (1991), p. 381-404

<http://www.numdam.org/item?id=JTNB_1991_3_2_381_0>

(C) Université Bordeaux 1, 1991, tous droits réservés.

L'accès aux archives de la revue "Séminaire de Théorie des Nombres de Bordeaux » (http://jtnb.cedram.org/) implique l'accord avec les conditions générales d'utilisation (http://www.numdam.org/conditions). Toute utilisation commerciale ou impression systématique est constitutive d'une infraction pénale. Toute copie ou impression de ce fichier doit contenir la présente mention de copyright.

\section{Numdam}

Article numérisé dans le cadre du programme

Numérisation de documents anciens mathématiques

http://www.numdam.org/ 
Séminaire de Théorie des Nombres,

Bordeaux 3 (1991), 381-404

\title{
Corps sextiques primitifs (IV)
}

\author{
par Michel OLIVIER
}

Nous terminons la publication des tables de corps sextiques (cf. vol.1, fasc.1 (1989), p.205-250, vol.2, fasc.2 (1990), p.49-102, et vol.3, fasc.1 (1991), p.201-245, in Séminaire de Théorie des Nombres de Bordeaux), par les quatre tables (une par signature) de corps sextiques primitifs. Les méthodes algorithmiques utilisées pour construire ces tables sont décrites dans l'article "corps sextiques primitifs" (in Annales de l'Institut Fourier, vol. 40, fasc. 4, 1990, p. 757-767).

Chacune des quatre tables (à l'exception de la table des corps totalement réels) contient les 200 premiers corps sextiques classés par valeur absolue croissante du discriminant. Des tables plus étendues sont disponibles sur support papier ou sur support magnétique (fichier source $\mathrm{T}_{\mathrm{E}} \mathrm{X}$ ).

Les tables contiennent les données suivantes : de gauche à droite,

le discriminant $d_{K}$ du corps sextique $K$, la factorisation de $d_{K}$.

le type $(*)$ de la clôture galoisienne de $K / \mathbb{Q}$,

le facteur parasite $f$ tel que $d_{P}=f^{2} d_{K}$.

un polynôme $P(X)$ définissant $K / \mathbb{Q}$.

(*) Les types possibles pour la clôture galoisienne de $K / \mathbb{Q}$ sont:

$$
A 5, A 6, S 5, S 6 \text {. }
$$

Manuscrit reçu le 10 mai 1989. 


\section{SIgNature $(6,0)$ PRIMITIF}

LISTE DES CORPS DE NOMBRES, DE SIGNATURE (6,0), DE DEGRÉ 6, DE DISCRIMINANT EN VALEUR ABSOLUE INFÉRIEUR OU ÉGAL À 10000000, PRIMITIFS.

Cette table contient :

0 corps de type A5

0 corps de type $\mathbf{A} 6$

0 corps de type $\mathrm{S} 5$

177 corps de type S6

Soit au total :

177 corps dans cette signature.

$\begin{array}{lllll}592661 & 592661 & \text { S6 } & 1 & x^{6}+2 x^{5}-5 x^{4}-4 x^{3}+5 x^{2}+x-1 \\ 1134389 & 1134389 & \text { S6 } & 1 & x^{6}+x^{5}-6 x^{4}-7 x^{3}+5 x^{2}+6 x+1 \\ 1202933 & 79 \times 15227 & \text { S6 } & 1 & x^{6}-9 x^{4}-9 x^{3}+4 x^{2}+5 x+1 \\ 1541581 & 1541581 & \text { S6 } & 1 & x^{6}-8 x^{4}+18 x^{2}+x-11 \\ 1868969 & 107 \times 17467 & \text { S6 } & 1 & x^{6}-10 x^{4}+3 x^{3}+9 x^{2}-6 x+1 \\ 2286997 & 349 \times 6553 & \text { S6 } & 1 & x^{6}-10 x^{4}-6 x^{3}+22 x^{2}+17 x-5 \\ 2323397 & 2323397 & \text { S6 } & 1 & x^{6}+3 x^{5}-9 x^{4}-42 x^{3}-45 x^{2}-10 x+1 \\ 2460365 & 5 \times 263 \times 1871 & \text { S6 } & 1 & x^{6}+x^{5}-8 x^{4}-4 x^{3}+17 x^{2}-x-1 \\ 2495261 & 199 \times 12539 & \text { S6 } & 1 & x^{6}+x^{5}-6 x^{4}-6 x^{3}+6 x^{2}+6 x+1 \\ 2501557 & 769 \times 3253 & \text { S6 } & 1 & x^{6}+2 x^{5}-9 x^{4}-8 x^{3}+23 x^{2}+7 x-17 \\ 2565429 & 3 \times 855143 & \text { S6 } & 1 & x^{6}+2 x^{5}-11 x^{4}+x^{3}+20 x^{2}-15 x+3 \\ 2661761 & 41 \times 64921 & \text { S6 } & 1 & x^{6}+x^{5}-10 x^{4}+x^{3}+13 x^{2}+3 x-1 \\ 2666432 & 2^{6} \times 61 \times 683 & \text { S6 } & 1 & x^{6}+2 x^{5}-6 x^{4}-4 x^{3}+7 x^{2}+2 x-1\end{array}$




\begin{tabular}{|c|c|c|c|c|}
\hline 2782261 & $643 \times 4327$ & S6 & 1 & $x^{6}-8 x^{4}+3 x^{3}+16 x^{2}-8 x-5$ \\
\hline 2812877 & $23 \times 122299$ & S6 & 1 & $x^{6}+3 x^{5}-4 x^{4}-14 x^{3}+4 x^{2}+16 x-1$ \\
\hline 2847089 & $7 \times 101 \times 4027$ & S6 & 1 & $x^{6}+3 x^{5}-3 x^{4}-12 x^{3}-3 x^{2}+5 x+2$ \\
\hline 3072812 & $2^{2} \times 768203$ & S6 & 1 & $x^{6}+x^{5}-6 x^{4}-4 x^{3}+7 x^{2}+2 x-2$ \\
\hline 3086597 & $383 \times 8059$ & S6 & 1 & $x^{6}+2 x^{5}-8 x^{4}-22 x^{3}-10 x^{2}+7 x+3$ \\
\hline 3151861 & 3151861 & S6 & 1 & $x^{6}+x^{5}-12 x^{4}-14 x^{3}+23 x^{2}+35 x+9$ \\
\hline 3184733 & 3184733 & S6 & 1 & $x^{6}+2 x^{5}-11 x^{4}-13 x^{3}+42 x^{2}+13 x-41$ \\
\hline 3319769 & $101 \times 32869$ & s6 & 1 & $x^{6}+3 x^{5}-4 x^{4}-11 x^{3}+7 x^{2}+9 x-4$ \\
\hline 3389609 & $59 \times 73 \times 787$ & S6 & 1 & $x^{6}+3 x^{5}-3 x^{4}-10 x^{3}+x^{2}+7 x+2$ \\
\hline 3477989 & $41^{2} \times 2069$ & S6 & 1 & $x^{6}+x^{5}-8 x^{4}-4 x^{3}+8 x^{2}+6 x+1$ \\
\hline 3486377 & $17 \times 205081$ & S6 & 1 & $x^{6}-6 x^{4}-x^{3}+7 x^{2}+x-1$ \\
\hline 3697873 & $997 \times 3709$ & S6 & 1 & $x^{6}+3 x^{5}-4 x^{4}-17 x^{3}-9 x^{2}+5 x+2$ \\
\hline 3706688 & $2^{6} \times 57917$ & S6 & 1 & $x^{6}-8 x^{4}+4 x^{3}+13 x^{2}-8 x-1$ \\
\hline 3728437 & $1913 \times 1949$ & S6 & 1 & $x^{6}+x^{5}-7 x^{4}-4 x^{3}+9 x^{2}+2 x-3$ \\
\hline 3822093 & $3^{3} \times 11 \times 17 \times 757$ & S6 & 1 & $x^{6}+3 x^{5}-4 x^{4}-10 x^{3}+3 x^{2}+5 x+1$ \\
\hline 3928381 & 3928381 & S6 & 1 & $x^{6}-8 x^{4}-2 x^{3}+6 x^{2}+x-1$ \\
\hline 1086536 & $2^{3} \times 510817$ & S6 & 1 & $x^{6}+x^{5}-11 x^{4}+3 x^{3}+13 x^{2}-10 x+2$ \\
\hline$\$ 125937$ & $139 \times 29683$ & s6 & 1 & $x^{6}+2 x^{5}-8 x^{4}-14 x^{3}+9 x^{2}+7 x+1$ \\
\hline 4170688 & $2^{6} \times 65167$ & s6 & 1 & $x^{6}-10 x^{4}-10 x^{3}+9 x^{2}+8 x-3$ \\
\hline 4181517 & $3^{3} \times 154871$ & S6 & 1 & $x^{6}+2 x^{5}-8 x^{4}-8 x^{3}+4 x^{2}+5 x+1$ \\
\hline 4224413 & $1193 \times 3541$ & S6 & 1 & $x^{6}-9 x^{4}+3 x^{3}+6 x^{2}-x-1$ \\
\hline 4254689 & $19 \times 61 \times 3671$ & S6 & 1 & $x^{6}-10 x^{4}-9 x^{3}+12 x^{2}+15 x+4$ \\
\hline 4274669 & $7 \times 610667$ & S6 & 1 & $x^{6}+x^{5}-8 x^{4}-8 x^{3}+13 x^{2}+7 x-7$ \\
\hline 4308028 & $2^{2} \times 79 \times 13633$ & S6 & 1 & $x^{6}+x^{5}-6 x^{4}-4 x^{3}+9 x^{2}+2 x-2$ \\
\hline 4383253 & $7 \times 409 \times 1531$ & S6 & 1 & $x^{6}+3 x^{5}-7 x^{4}-10 x^{3}+20 x^{2}-9 x+1$ \\
\hline 4418197 & $7 \times 631171$ & S6 & 1 & $x^{6}-13 x^{4}-17 x^{3}+10 x^{2}+21 x+7$ \\
\hline 4443861 & $3 \times 97 \times 15271$ & S6 & 1 & $x^{6}+x^{5}-8 x^{4}-8 x^{3}+10 x^{2}+12 x+3$ \\
\hline 4448597 & $47 \times 94651$ & S6 & 1 & $x^{6}+x^{5}-7 x^{4}-10 x^{3}+2 x^{2}+5 x+1$ \\
\hline 4537077 & $3 \times 947 \times 1597$ & S6 & 1 & $x^{6}+x^{5}-9 x^{4}+20 x^{2}-15 x+3$ \\
\hline 4601153 & 4601153 & S6 & 1 & $x^{6}+3 x^{5}-8 x^{4}-19 x^{3}+27 x^{2}+31 x-37$ \\
\hline 4733829 & $3^{3} \times 175327$ & S6 & 1 & $x^{6}-10 x^{4}-x^{3}+18 x^{2}-10 x+1$ \\
\hline 4755281 & 4755281 & S6 & 1 & $x^{6}+x^{5}-10 x^{4}-x^{3}+29 x^{2}-19 x-2$ \\
\hline 4758548 & $2^{2} \times 1189637$ & S6 & 1 & $x^{6}-10 x^{4}-3 x^{3}+16 x^{2}-5 x-1$ \\
\hline 4823921 & $311 \times 15511$ & S6 & 1 & $x^{6}+x^{5}-12 x^{4}-x^{3}+13 x^{2}+7 x+1$ \\
\hline 4838537 & $11 \times 439867$ & S6 & 1 & $x^{6}+3 x^{5}-3 x^{4}-11 x^{3}+x^{2}+9 x+1$ \\
\hline 4918997 & 4918997 & S6 & 1 & $x^{6}+2 x^{5}-8 x^{4}-24 x^{3}-16 x^{2}+x+1$ \\
\hline 5030996 & $2^{2} \times 1257749$ & S6 & 1 & $x^{6}+3 x^{5}-8 x^{4}-21 x^{3}+26 x^{2}+36 x-38$ \\
\hline 5061656 & $2^{3} \times 23 \times 27509$ & S6 & 1 & $x^{6}+x^{5}-12 x^{4}-4 x^{3}+14 x^{2}+9 x+1$ \\
\hline 5090861 & $67 \times 75983$ & S6 & 1 & $x^{6}+3 x^{5}-6 x^{4}-19 x^{3}-x^{2}+16 x+7$ \\
\hline 5101781 & 5101781 & s6 & 1 & $x^{6}+3 x^{5}-9 x^{4}-16 x^{3}+28 x^{2}+21 x-29$ \\
\hline 5160733 & 5160733 & S6 & 1 & $x^{6}+3 x^{5}-8 x^{4}-30 x^{3}-24 x^{2}-4 x+1$ \\
\hline
\end{tabular}




\begin{tabular}{|c|c|c|c|c|}
\hline 5224841 & $23 \times 227167$ & S6 & 1 & $x^{6}+2 x^{5}-5 x^{4}-7 x^{3}+7 x^{2}+2 x-1$ \\
\hline 5279033 & 5279033 & S6 & 1 & $x^{6}-8 x^{4}+4 x^{3}+9 x^{2}-x-1$ \\
\hline 5398157 & $1697 \times 3181$ & S6 & 1 & $x^{6}+2 x^{5}-8 x^{4}-7 x^{3}+18 x^{2}-8 x+1$ \\
\hline 5402093 & 5402093 & S6 & 1 & $x^{6}+3 x^{5}-4 x^{4}-18 x^{3}-8 x^{2}+12 x+7$ \\
\hline 5431612 & $2^{2} \times 97 \times 13999$ & s6 & 1 & $x^{6}+x^{5}-10 x^{4}+2 x^{3}+15 x^{2}-2 x-6$ \\
\hline 5489889 & $3 \times 1829963$ & S6 & 1 & $x^{6}+x^{5}-10 x^{4}+3 x^{3}+13 x^{2}-3 x-2$ \\
\hline 5611169 & 5611169 & S6 & 1 & $x^{6}+x^{5}-11 x^{4}-7 x^{3}+20 x^{2}-9 x+1$ \\
\hline 5625337 & $487 \times 11551$ & S6 & 1 & $x^{6}+3 x^{5}-3 x^{4}-12 x^{3}-x^{2}+9 x+2$ \\
\hline 5689408 & $2^{6} \times 88897$ & S6 & 1 & $x^{6}-7 x^{4}-2 x^{3}+11 x^{2}+4 x-1$ \\
\hline 5699033 & 5699033 & S6 & 1 & $x^{6}+2 x^{5}-10 x^{4}-9 x^{3}+19 x^{2}-8 x+1$ \\
\hline 5740564 & $2^{2} \times 1435141$ & S6 & 1 & $x^{6}+2 x^{5}-8 x^{4}-3 x^{3}+6 x^{2}+x-1$ \\
\hline 5748973 & 5748973 & S6 & 1 & $x^{6}+x^{5}-6 x^{4}-4 x^{3}+8 x^{2}+4 x-1$ \\
\hline 5861477 & $2069 \times 2833$ & S6 & 1 & $x^{6}+2 x^{5}-5 x^{4}-10 x^{3}+3 x^{2}+7 x+1$ \\
\hline 5869904 & $2^{4} \times 366869$ & S6 & 1 & $x^{6}+x^{5}-8 x^{4}-8 x^{3}+16 x^{2}+16 x-2$ \\
\hline 5926169 & 5926169 & S6 & 1 & $x^{6}+2 x^{5}-5 x^{4}-7 x^{3}+7 x^{2}+3 x-2$ \\
\hline 5972789 & $137 \times 43597$ & S6 & 1 & $x^{6}+x^{5}-8 x^{4}-2 x^{3}+16 x^{2}-8 x+1$ \\
\hline 6018905 & $5 \times 227 \times 5303$ & S6 & 1 & $x^{6}+2 x^{5}-11 x^{4}-13 x^{3}+31 x^{2}-7 x-4$ \\
\hline 6084425 & $5^{2} \times 199 \times 1223$ & S6 & 1 & $x^{6}+3 x^{5}-5 x^{4}-21 x^{3}-7 x^{2}+21 x+13$ \\
\hline 6127252 & $2^{2} \times 1531813$ & S6 & 1 & $x^{6}+x^{5}-6 x^{4}-5 x^{3}+8 x^{2}+4 x-2$ \\
\hline 6139004 & $2^{2} \times 1534751$ & S6 & 1 & $x^{6}-7 x^{4}-3 x^{3}+7 x^{2}+x-1$ \\
\hline 6241669 & $7^{2} \times 17 \times 59 \times 127$ & S6 & 1 & $x^{6}+x^{5}-8 x^{4}-10 x^{3}+7 x^{2}+11 x+3$ \\
\hline 6273133 & $53 \times 118361$ & S6 & 1 & $x^{6}+2 x^{5}-5 x^{4}-7 x^{3}+4 x^{2}+3 x-1$ \\
\hline 6293081 & 6293081 & S6 & 1 & $x^{6}+3 x^{5}-3 x^{4}-13 x^{3}-5 x^{2}+3 x+1$ \\
\hline 6460157 & $11 \times 587287$ & S6 & 1 & $x^{6}+3 x^{5}-3 x^{4}-10 x^{3}+2 x^{2}+7 x+1$ \\
\hline 6550837 & $23 \times 284819$ & S6 & 1 & $x^{6}+3 x^{5}-8 x^{4}-35 x^{3}-33 x^{2}-2 x+5$ \\
\hline 6554149 & $7 \times 23 \times 40709$ & S6 & 1 & $x^{6}+2 x^{5}-11 x^{4}-24 x^{3}+25 x^{2}+69 x+23$ \\
\hline 6557081 & 6557081 & S6 & 1 & $x^{6}+2 x^{5}-11 x^{4}-21 x^{3}+31 x^{2}+55 x-4$ \\
\hline 6607192 & $2^{5} \times 53 \times 15583$ & S6 & 1 & $x^{6}+3 x^{5}-3 x^{4}-11 x^{3}-x^{2}+6 x+2$ \\
\hline 6609217 & $179 \times 36923$ & S6 & 1 & $x^{6}-10 x^{4}+13 x^{2}-7 x+1$ \\
\hline 6754732 & $2^{2} \times 23 \times 73421$ & s6 & 1 & $x^{6}+x^{5}-9 x^{4}-9 x^{3}+7 x^{2}+6 x+1$ \\
\hline 6799312 & $2^{4} \times 13 \times 97 \times 337$ & S6 & 1 & $x^{6}+3 x^{5}-8 x^{4}-9 x^{3}+4 x^{2}+5 x+1$ \\
\hline 6848593 & $643 \times 10651$ & S6 & 1 & $x^{6}+3 x^{5}-7 x^{4}-29 x^{3}-12 x^{2}+34 x+27$ \\
\hline 6856697 & 6856697 & S6 & 1 & $x^{6}+3 x^{5}-3 x^{4}-12 x^{3}+8 x-1$ \\
\hline 6869477 & $769 \times 8933$ & S6 & 1 & $x^{6}+x^{5}-12 x^{4}-15 x^{3}+33 x^{2}+54 x+19$ \\
\hline 6955589 & 6955589 & S6 & 1 & $x^{6}+3 x^{5}-4 x^{4}-12 x^{3}+3 x^{2}+11 x+3$ \\
\hline 6968756 & $2^{2} \times 241 \times 7229$ & S6 & 1 & $x^{6}+2 x^{5}-11 x^{4}-x^{3}+12 x^{2}-2 x-2$ \\
\hline 6995813 & $11 \times 635983$ & S6 & 1 & $x^{6}+x^{5}-7 x^{4}-4 x^{3}+6 x^{2}+x-1$ \\
\hline 6999257 & $17 \times 411721$ & S6 & 1 & $x^{6}+x^{5}-11 x^{4}+7 x^{3}+6 x^{2}-2 x-1$ \\
\hline 7050937 & $17 \times 241 \times 1721$ & S6 & 1 & $x^{6}-7 x^{4}+2 x^{3}+8 x^{2}-x-2$ \\
\hline 7108233 & $3 \times 11 \times 47 \times 4583$ & S6 & 1 & $x^{6}+2 x^{5}-11 x^{4}-11 x^{3}+32 x^{2}-18 x+3$ \\
\hline 7229081 & $263 \times 27487$ & S6 & 1 & $x^{6}-9 x^{4}-2 x^{3}+22 x^{2}+9 x-8$ \\
\hline
\end{tabular}




\begin{tabular}{|c|c|c|c|c|}
\hline 7281497 & $31^{2} \times 7577$ & S6 & 1 & $x^{6}+2 x^{5}-8 x^{4}-15 x^{3}+12 x^{2}+27 x+10$ \\
\hline 7313969 & $13 \times 562613$ & S6 & 1 & $x^{6}-8 x^{4}-3 x^{3}+14 x^{2}+5 x-4$ \\
\hline 7319833 & $613 \times 11941$ & S6 & 1 & $x^{6}+3 x^{5}-3 x^{4}-13 x^{3}-2 x^{2}+10 x+3$ \\
\hline 7342612 & $2^{2} \times 23 \times 79811$ & S6 & 1 & $x^{6}+3 x^{5}-8 x^{4}-37 x^{3}-40 x^{2}-12 x-1$ \\
\hline 7383296 & $2^{8} \times 151 \times 191$ & S6 & 1 & $x^{6}+x^{5}-9 x^{4}-10 x^{3}+13 x^{2}+7 x-5$ \\
\hline 7432373 & $13 \times 571721$ & S6 & 1 & $x^{6}+x^{5}-12 x^{4}-26 x^{3}-10 x^{2}+7 x+2$ \\
\hline 7454269 & 7454269 & S6 & 1 & $x^{6}+3 x^{5}-4 x^{4}-15 x^{3}-x^{2}+12 x+5$ \\
\hline 7520053 & $373 \times 20161$ & S6 & 1 & $x^{6}+2 x^{5}-8 x^{4}-5 x^{3}+16 x^{2}-8 x+1$ \\
\hline 7526677 & $43 \times 175039$ & S6 & 1 & $x^{6}+2 x^{5}-11 x^{4}-10 x^{3}+5 x^{2}+3 x-1$ \\
\hline 7563989 & 7563989 & S6 & 1 & $x^{6}+x^{5}-12 x^{4}-14 x^{3}+4 x+1$ \\
\hline 7599997 & $821 \times 9257$ & S6 & 1 & $x^{6}+x^{5}-8 x^{4}-13 x^{3}-x^{2}+4 x+1$ \\
\hline 7606541 & $1471 \times 5171$ & S6 & 1 & $x^{6}+3 x^{5}-5 x^{4}-22 x^{3}-15 x^{2}+2 x+1$ \\
\hline 7633856 & $2^{6} \times 181 \times 659$ & S6 & 1 & $x^{6}-13 x^{4}-4 x^{3}+24 x^{2}-12 x+1$ \\
\hline 7646825 & $5^{2} \times 305873$ & S6 & 1 & $x^{6}+2 x^{5}-11 x^{4}-28 x^{3}+10 x^{2}+65 x+40$ \\
\hline 7649984 & $2^{6} \times 23 \times 5197$ & S6 & 1 & $x^{6}-13 x^{4}-16 x^{3}+22 x^{2}+44 x+17$ \\
\hline 7693877 & $17 \times 101 \times 4481$ & S6 & 1 & $x^{6}-10 x^{4}-2 x^{3}+28 x^{2}+5 x-23$ \\
\hline 7735165 & $5 \times 163 \times 9491$ & S6 & 1 & $x^{6}+3 x^{5}-8 x^{4}-24 x^{3}-8 x^{2}+10 x+5$ \\
\hline 7742321 & 7742321 & S6 & 1 & $x^{6}+2 x^{5}-8 x^{4}-21 x^{3}-9 x^{2}+5 x+1$ \\
\hline 7764889 & $11 \times 705899$ & S6 & 1 & $x^{6}-9 x^{4}-5 x^{3}+17 x^{2}+11 x-4$ \\
\hline 7783241 & 7783241 & S6 & 1 & $x^{6}+2 x^{5}-5 x^{4}-9 x^{3}+5 x^{2}+6 x-1$ \\
\hline 7836289 & $41 \times 131 \times 1459$ & S6 & 1 & $x^{6}+2 x^{5}-8 x^{4}-20 x^{3}-5 x^{2}+7 x-1$ \\
\hline 7885677 & $3 \times 2628559$ & S6 & 1 & $x^{6}+2 x^{5}-5 x^{4}-8 x^{3}+5 x^{2}+3 x-1$ \\
\hline 7905501 & $3^{2} \times 19 \times 83 \times 557$ & S6 & 1 & $x^{6}-7 x^{4}-2 x^{3}+11 x^{2}+3 x-3$ \\
\hline 8047141 & 8047141 & s6 & 1 & $x^{6}+2 x^{5}-8 x^{4}-16 x^{3}+12 x^{2}+33 x+13$ \\
\hline 8086336 & $2^{6} \times 126349$ & S6 & 1 & $x^{6}-9 x^{4}-4 x^{3}+14 x^{2}+2 x-5$ \\
\hline 8246037 & $3 \times 17^{2} \times 9511$ & S6 & 1 & $x^{6}+x^{5}-8 x^{4}-7 x^{3}+17 x^{2}+12 x-7$ \\
\hline 8256989 & $13 \times 13 \times 14771$ & S6 & 1 & $x^{6}+x^{5}-10 x^{4}-4 x^{3}+14 x^{2}+2 x-5$ \\
\hline 8263504 & $24 \times 516469$ & S6 & 1 & $x^{6}+3 x^{5}-4 x^{4}-17 x^{3}-4 x^{2}+17 x+9$ \\
\hline 8283692 & $2^{2} \times 17 \times 43 \times 2833$ & S6 & 1 & $x^{6}+3 x^{5}-3 x^{4}-11 x^{3}+x^{2}+8 x+2$ \\
\hline 8284569 & $3 \times 199 \times 13877$ & S6 & 1 & $x^{6}+2 x^{5}-8 x^{4}-17 x^{3}+4 x^{2}+11 x+2$ \\
\hline 8287853 & $7 \times 587 \times 2017$ & S6 & 1 & $x^{6}-10 x^{4}-7 x^{3}+22 x^{2}+26 x+5$ \\
\hline 8357033 & 8357033 & S6 & 1 & $x^{6}+3 x^{5}-4 x^{4}-13 x^{3}+x^{2}+9 x-1$ \\
\hline 8479181 & $113 \times 75037$ & S6 & 1 & $x^{6}+x^{5}-10 x^{4}-11 x^{3}+11 x^{2}+8 x-5$ \\
\hline 8496848 & $2^{4} \times 47 \times 11299$ & S6 & 1 & $x^{6}+x^{5}-6 x^{4}-5 x^{3}+6 x^{2}+3 x-1$ \\
\hline 8513872 & $2 \times 17 \times 113 \times 277$ & s6 & 1 & $x^{6}+x^{5}-6 x^{4}-3 x^{3}+8 x^{2}+x-1$ \\
\hline 8517401 & 8517401 & S6 & 1 & $x^{6}+x^{5}-10 x^{4}-7 x^{3}+31 x^{2}+11 x-31$ \\
\hline 8592653 & 8592653 & s6 & 1 & $x^{6}+3 x^{5}-4 x^{4}-16 x^{3}-3 x^{2}+15 x+7$ \\
\hline 8606077 & 8606077 & S6 & 1 & $x^{6}+x^{5}-10 x^{4}-8 x^{3}+27 x^{2}+11 x-23$ \\
\hline 8611796 & $2^{2} \times 2152949$ & S6 & 1 & $x^{6}+2 x^{5}-5 x^{4}-7 x^{3}+6 x^{2}+4 x-2$ \\
\hline 8613337 & $563 \times 15299$ & S6 & 1 & $x^{6}+2 x^{5}-8 x^{4}-15 x^{3}+13 x^{2}+16 x-11$ \\
\hline 8639957 & $2711 \times 3187$ & S6 & 1 & $x^{6}+3 x^{5}-4 x^{4}-16 x^{3}-3 x^{2}+10 x+1$ \\
\hline
\end{tabular}




\begin{tabular}{|c|c|c|c|c|}
\hline 8758061 & $13 \times 383 \times 1759$ & S6 & 1 & $x^{6}+3 x^{5}-8 x^{4}-36 x^{3}-31 x^{2}+7 x+11$ \\
\hline 8769809 & 8769809 & S6 & 1 & $x^{6}+3 x^{5}-5 x^{4}-17 x^{3}+3 x^{2}+19 x+7$ \\
\hline 8793749 & 8793749 & S6 & 1 & $x^{6}+2 x^{5}-6 x^{4}-7 x^{3}+6 x^{2}+6 x+1$ \\
\hline 8815673 & $17 \times 641 \times 809$ & S6 & 1 & $x^{6}+3 x^{5}-7 x^{4}-23 x^{3}+x^{2}+17 x+1$ \\
\hline 8985001 & 8985001 & S6 & 1 & $x^{6}+3 x^{5}-8 x^{4}-29 x^{3}+5 x^{2}+67 x+44$ \\
\hline 9011589 & $3 \times 3003863$ & S6 & 1 & $x^{6}+x^{5}-12 x^{4}+12 x^{3}+3 x^{2}-5 x+1$ \\
\hline 9059636 & $2^{2} \times 197 \times 11497$ & S6 & 1 & $x^{6}+2 x^{5}-11 x^{4}-33 x^{3}-20 x^{2}+4 x+2$ \\
\hline 9111769 & $521 \times 17489$ & S6 & 1 & $x^{6}+2 x^{5}-11 x^{4}-5 x^{3}+27 x^{2}-2 x-13$ \\
\hline 9114573 & $3 \times 13 \times 103 \times 2269$ & S6 & 1 & $x^{6}+2 x^{5}-8 x^{4}-4 x^{3}+6 x^{2}+x-1$ \\
\hline 9117749 & $53 \times 71 \times 2423$ & s6 & 1 & $x^{6}+2 x^{5}-8 x^{4}-15 x^{3}+16 x^{2}+28 x-1$ \\
\hline 9125696 & $2^{6} \times 142589$ & 56 & 1 & $x^{6}+3 x^{5}-3 x^{4}-12 x^{3}+10 x+2$ \\
\hline 9129208 & $2^{3} \times 11^{2} \times 9431$ & S6 & 2 & $x^{6}+x^{5}-12 x^{4}-2 x^{3}+40 x^{2}-25 x+1$ \\
\hline 9202801 & $127 \times 233 \times 311$ & S6 & 1 & $x^{6}+x^{5}-8 x^{4}-9 x^{3}+9 x^{2}+7 x-4$ \\
\hline 9262328 & $2^{3} \times 1157791$ & S6 & 1 & $x^{6}+x^{5}-7 x^{4}-x^{3}+9 x^{2}-1$ \\
\hline 9273877 & $313 \times 29629$ & S6 & 1 & $x^{6}+2 x^{5}-10 x^{4}-12 x^{3}+30 x^{2}+3 x-13$ \\
\hline 9326293 & $23 \times 405491$ & S6 & 1 & $x^{6}+3 x^{5}-8 x^{4}-23 x^{3}+9 x^{2}+46 x+23$ \\
\hline 9349689 & $3 \times 3116563$ & S6 & 1 & $x^{6}+x^{5}-7 x^{4}-7 x^{3}+11 x^{2}+9 x-3$ \\
\hline 9372736 & $2^{6} \times 146449$ & S6 & 1 & $x^{6}+2 x^{5}-5 x^{4}-6 x^{3}+6 x^{2}+2 x-1$ \\
\hline 9378277 & 9378277 & S6 & 1 & $x^{6}-10 x^{4}-6 x^{3}+22 x^{2}+25 x+7$ \\
\hline 9468304 & $2 \times 151 \times 3919$ & S6 & 1 & $x^{6}+x^{5}-7 x^{4}-8 x^{3}+7 x^{2}+7 x+1$ \\
\hline 9502656 & $2^{6} \times 3 \times 43 \times 1151$ & S6 & 1 & $x^{6}-7 x^{4}-2 x^{3}+8 x^{2}-1$ \\
\hline 9526933 & $13 \times 732841$ & S6 & 1 & $x^{6}+x^{5}-10 x^{4}-14 x^{3}+6 x^{2}+8 x-3$ \\
\hline 9543232 & $2^{6} \times 149113$ & s6 & 4 & $x^{6}+x^{5}-10 x^{4}+x^{3}+8 x^{2}-x-1$ \\
\hline 9596117 & 9596117 & S6 & 1 & $x^{6}-10 x^{4}-8 x^{3}+19 x^{2}+23 x+4$ \\
\hline 9627061 & 9627061 & S6 & 1 & $x^{6}+x^{5}-8 x^{4}-6 x^{3}+11 x^{2}+7 x+1$ \\
\hline 9649877 & 9649877 & S6 & 1 & $x^{6}+2 x^{5}-11 x^{4}-26 x^{3}+21 x^{2}+83 x+49$ \\
\hline 9652257 & $3^{3} \times 389 \times 919$ & S6 & 1 & $x^{6}+2 x^{5}-11 x^{4}-5 x^{3}+37 x^{2}-30 x+7$ \\
\hline 9711596 & $2^{2} \times 2427899$ & S6 & 1 & $x^{6}+3 x^{5}-8 x^{4}-4 x^{3}+6 x^{2}+x-1$ \\
\hline 9742016 & $2^{6} \times 152219$ & S6 & 1 & $x^{6}+2 x^{5}-8 x^{4}-4 x^{3}+13 x^{2}+2 x-5$ \\
\hline 9761821 & $23 \times 37 \times 11471$ & S6 & 1 & $x^{6}+x^{5}-10 x^{4}-12 x^{3}+18 x^{2}+14 x-13$ \\
\hline 9772397 & $521 \times 18757$ & S6 & 1 & $x^{6}+3 x^{5}-8 x^{4}-26 x^{3}+10 x^{2}+38 x-13$ \\
\hline 9777728 & $2^{6} \times 152777$ & S6 & 1 & $x^{6}+2 x^{5}-10 x^{4}-6 x^{3}+25 x^{2}-16 x+3$ \\
\hline 9783113 & 9783113 & S6 & 1 & $x^{6}+x^{5}-12 x^{4}-13 x^{3}+31 x^{2}+19 x-26$ \\
\hline 9810092 & $2^{2} \times 2452523$ & S6 & 1 & $x^{6}+2 x^{5}-8 x^{4}-23 x^{3}-13 x^{2}+4 x+2$ \\
\hline 9811577 & 9811577 & S6 & 8 & $x^{6}+x^{5}-10 x^{4}-x^{3}+18 x^{2}-1$ \\
\hline 9855552 & $2^{6} \times 3 \times 7 \times 7333$ & S6 & 1 & $x^{6}+2 x^{5}-6 x^{4}-14 x^{3}+x^{2}+12 x+3$ \\
\hline 9921856 & $2^{6} \times 7 \times 22147$ & s6 & 1 & $x^{6}+2 x^{5}-11 x^{4}+7 x^{2}-1$ \\
\hline 9925933 & $149 \times 66617$ & S6 & 1 & $x^{6}+x^{5}-10 x^{4}+6 x^{3}+6 x^{2}-2 x-1$ \\
\hline 9944521 & $31 \times 320791$ & S6 & 1 & $x^{6}+2 x^{5}-11 x^{4}-7 x^{3}+41 x^{2}-25 x-2$ \\
\hline 9967801 & 9967801 & S6 & 1 & $x^{6}+x^{5}-9 x^{4}-5 x^{3}+6 x^{2}+2 x-1$ \\
\hline 9979841 & $359 \times 27799$ & S6 & 1 & $x^{6}-9 x^{4}-2 x^{3}+12 x^{2}+x-4$ \\
\hline
\end{tabular}




\section{SIGNATURE $(4,1)$ PRIMITIF}

LISTE DES CORPS DE NOMBRES, DE SIGNATURE (4,1), DE DEGRÉ 6,

DE DISCRIMINANT EN VALEUR ABSOLUE INFÉRIEUR OU ÉGAL À 748000 ,

PRIMITIFS.

Cette table contient :

0 corps de type A5

0 corps de type A6

0 corps de type $\mathrm{S} 5$

200 corps de type $\mathrm{S} 6$

Soit au total :

200 corps dans cette signature.

$\begin{array}{lllll}-92779 & -92779 & \text { S6 } & 1 & x^{6}-6 x^{4}-11 x^{3}-4 x^{2}+2 x+1 \\ -94363 & -197 \times 479 & \text { S6 } & 1 & x^{6}-2 x^{4}+2 x^{3}-3 x+1 \\ -104483 & -163 \times 641 & \text { S6 } & 1 & x^{6}+x^{5}-2 x^{4}-2 x^{3}-x^{2}+x+1 \\ -118987 & -11 \times 29 \times 373 & \text { S6 } & 1 & x^{6}+2 x^{5}-3 x^{4}-7 x^{3}-2 x^{2}-x-1 \\ -133787 & -353 \times 379 & \text { S6 } & 1 & x^{6}-4 x^{4}-2 x^{3}+2 x^{2}+3 x+1 \\ -161939 & -67 \times 2417 & \text { S6 } & 1 & x^{6}+2 x^{5}-2 x^{4}-7 x^{3}+6 x+1 \\ -195379 & -47 \times 4157 & \text { S6 } & 1 & x^{6}+3 x^{5}-2 x^{4}+x^{3}+9 x^{2}+6 x+1 \\ -209107 & -67 \times 3121 & \text { S6 } & 1 & x^{6}+3 x^{5}+4 x^{2}-10 x+1 \\ -214747 & -13 \times 16519 & \text { S6 } & 1 & x^{6}+x^{5}-3 x^{4}+2 x^{3}+5 x^{2}-6 x+1 \\ -215811 & -3^{3} \times 7993 & \text { S6 } & 1 & x^{6}+x^{5}-2 x^{4}+2 x^{3}+x^{2}-3 x+1 \\ -216587 & -7 \times 30941 & \text { S6 } & 1 & x^{6}+3 x^{5}-4 x^{4}-8 x^{3}+11 x^{2}-5 x+1 \\ -217024 & -2^{6} \times 3391 & \text { S6 } & 1 & x^{6}+2 x^{5}-2 x^{4}-6 x^{3}-x^{2}+4 x+1 \\ -225403 & -17 \times 13259 & \text { S6 } & 1 & x^{6}+3 x^{5}-2 x^{4}-8 x^{3}+3 x^{2}+5 x-1\end{array}$




\begin{tabular}{|c|c|}
\hline-228544 & $-2^{6} \times 3571$ \\
\hline-233003 & $-41 \times 5683$ \\
\hline-237179 & -237179 \\
\hline-238507 & $-19 \times 12553$ \\
\hline-242467 & -242467 \\
\hline-255179 & -255179 \\
\hline-255823 & $-109 \times 2347$ \\
\hline-257339 & -257339 \\
\hline-257344 & $-2^{6} \times 4021$ \\
\hline-259283 & $-251 \times 1033$ \\
\hline-264931 & -264931 \\
\hline-269395 & $-5 \times 7 \times 43 \times 179$ \\
\hline-295643 & $-239 \times 1237$ \\
\hline-302123 & -302123 \\
\hline-303619 & -303619 \\
\hline-304859 & $-83 \times 3673$ \\
\hline-306731 & $-17 \times 18043$ \\
\hline-311419 & -311419 \\
\hline-321527 & $-47 \times 6841$ \\
\hline-324619 & -324619 \\
\hline-326707 & -326707 \\
\hline-331787 & $-257 \times 1291$ \\
\hline-333019 & -333019 \\
\hline-337543 & -337543 \\
\hline-346167 & $-3^{3} \times 12821$ \\
\hline-348779 & -348779 \\
\hline-353383 & $-193 \times 1831$ \\
\hline-354103 & $-19 \times 18637$ \\
\hline-356443 & -356443 \\
\hline-358211 & $-7 \times 73 \times 701$ \\
\hline-360291 & $-3 \times 120097$ \\
\hline-361259 & $-241 \times 1499$ \\
\hline-365263 & $-23 \times 15881$ \\
\hline-367792 & $-2^{4} \times 127 \times 181$ \\
\hline-370219 & $-47 \times 7877$ \\
\hline-371464 & $-2^{3} \times 59 \times 787$ \\
\hline-375387 & $-3 \times 157 \times 797$ \\
\hline-382131 & $-3^{3} \times 14153$ \\
\hline-385467 & $-3 \times 128489$ \\
\hline-394299 & $-3^{2} \times 193 \times 227$ \\
\hline-400339 & -400339 \\
\hline
\end{tabular}

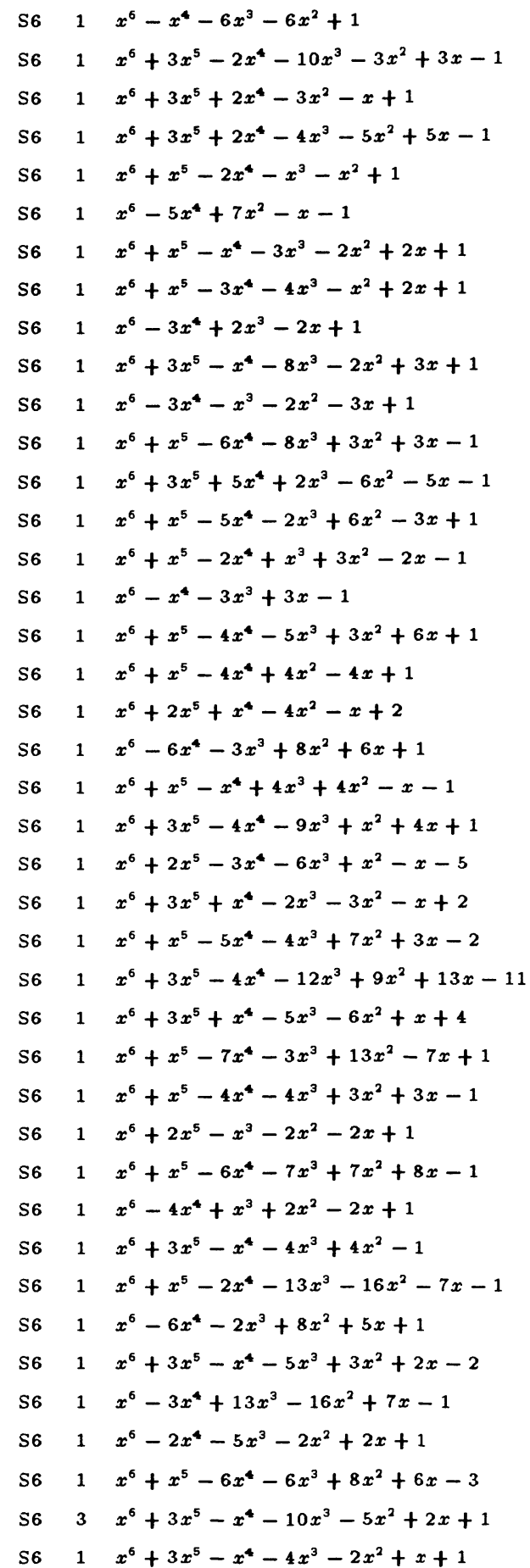




\begin{tabular}{|c|c|c|c|c|}
\hline-400648 & $-2^{3} \times 61 \times 821$ & S6 & 1 & $x^{6}+x^{5}-4 x^{4}-2 x^{3}+4 x^{2}-x-1$ \\
\hline-403947 & $-3^{4} \times 4987$ & s6 & 1 & $x^{6}+2 x^{5}-4 x^{4}-8 x^{3}+4 x^{2}+7 x+1$ \\
\hline-407767 & $-23 \times 17729$ & s6 & 1 & $x^{6}+3 x^{5}+4 x^{4}-5 x^{3}-5 x^{2}+5 x-1$ \\
\hline-417467 & $-229 \times 1823$ & s6 & 1 & $x^{6}+3 x^{5}-3 x^{3}+x^{2}-8 x-13$ \\
\hline-418867 & -418867 & S6 & 1 & $x^{6}-2 x^{4}-4 x^{3}+3 x+1$ \\
\hline-420743 & -420743 & S6 & 1 & $x^{6}+3 x^{5}-x^{4}-9 x^{3}-4 x^{2}+7 x+4$ \\
\hline-429327 & $-3^{3} \times 15901$ & S6 & 1 & $x^{6}-5 x^{4}-x^{3}+7 x^{2}+x-2$ \\
\hline-435283 & -435283 & S6 & 1 & $x^{6}+3 x^{5}-3 x^{4}-12 x^{3}+11 x+5$ \\
\hline-436927 & $-601 \times 727$ & S6 & 1 & $x^{6}+3 x^{5}+x^{4}-3 x^{3}-4 x^{2}-4 x-1$ \\
\hline-439216 & $-2^{4} \times 97 \times 283$ & S6 & 1 & $x^{6}+3 x^{5}-2 x^{4}-7 x^{3}+3 x+1$ \\
\hline-441411 & $-3 \times 147137$ & $\mathrm{~S} 6$ & 1 & $x^{6}-2 x^{4}-4 x^{3}+4 x^{2}+3 x-1$ \\
\hline-441751 & -441751 & S6 & 1 & $x^{6}+x^{5}-6 x^{4}-7 x^{3}+7 x^{2}+11 x+4$ \\
\hline-442443 & $-3 \times 147481$ & S6 & 1 & $x^{6}-7 x^{4}-x^{3}+14 x^{2}+3 x-7$ \\
\hline-443704 & $-2^{3} \times 37 \times 1499$ & S6 & 1 & $x^{6}+3 x^{5}+2 x^{4}-2 x^{2}-5 x-1$ \\
\hline-446827 & -446827 & S6 & 1 & $x^{6}-6 x^{4}-x^{3}+10 x^{2}+4 x-1$ \\
\hline-448351 & -448351 & S6 & 1 & $x^{6}+2 x^{5}-x^{4}+x^{3}+9 x^{2}+6 x+1$ \\
\hline-449107 & -449107 & S6 & 1 & $x^{6}-6 x^{4}+2 x^{3}+10 x^{2}-5 x-1$ \\
\hline-450124 & $-2^{2} \times 43 \times 2617$ & S6 & 1 & $x^{6}+3 x^{5}-2 x^{4}-9 x^{3}+2 x^{2}+6 x-2$ \\
\hline-454583 & $-233 \times 1951$ & S6 & 1 & $x^{6}+x^{5}-6 x^{4}-7 x^{3}+3 x^{2}+x-1$ \\
\hline-455771 & $-157 \times 2903$ & s6 & 1 & $x^{6}+x^{5}-7 x^{4}-8 x^{3}+3 x+1$ \\
\hline-459383 & -459383 & S6 & 1 & $x^{6}+3 x^{5}+3 x^{4}+3 x^{3}-4 x^{2}-21 x-16$ \\
\hline-463471 & $-17 \times 137 \times 199$ & S6 & 1 & $x^{6}+3 x^{5}-7 x^{3}-3 x^{2}+5 x-1$ \\
\hline-464483 & -464483 & S6 & 1 & $x^{6}+2 x^{5}-4 x^{4}-10 x^{3}+7 x+1$ \\
\hline-467939 & $-37 \times 12647$ & S6 & 1 & $x^{6}+2 x^{5}+6 x^{4}-x^{3}-10 x^{2}-6 x-1$ \\
\hline-472403 & $-433 \times 1091$ & S6 & 1 & $x^{6}+x^{5}-5 x^{4}-4 x^{3}+6 x^{2}-x-7$ \\
\hline-481979 & $-79 \times 6101$ & S6 & 1 & $x^{6}+3 x^{5}-2 x^{4}-8 x^{3}-2 x-1$ \\
\hline-483136 & $-2^{6} \times 7549$ & S6 & 1 & $x^{6}+6 x^{3}+x^{2}-4 x+1$ \\
\hline-488059 & $-11 \times 13 \times 3413$ & S6 & 1 & $x^{6}+3 x^{5}+2 x^{4}+2 x^{3}+4 x^{2}-1$ \\
\hline-493211 & -493211 & S6 & 1 & $x^{6}-5 x^{4}-x^{3}+8 x^{2}+x-3$ \\
\hline-493783 & $-29 \times 17027$ & S6 & 1 & $x^{6}+x^{5}-7 x^{4}-11 x^{3}+8 x^{2}+25 x+14$ \\
\hline-494231 & $-71 \times 6961$ & S6 & 1 & $x^{6}-7 x^{4}+14 x^{2}-x-8$ \\
\hline-495547 & $-13 \times 38119$ & S6 & 1 & $x^{6}+3 x^{5}-3 x^{4}-10 x^{3}+4 x^{2}+7 x-1$ \\
\hline-497883 & $-3 \times 165961$ & S6 & 1 & $x^{6}-5 x^{4}-2 x^{3}+7 x^{2}+3 x-3$ \\
\hline-498107 & $-149 \times 3343$ & S6 & 1 & $x^{6}+3 x^{5}-2 x^{4}-12 x^{3}-11 x^{2}-5 x-1$ \\
\hline-504443 & $-67 \times 7529$ & S6 & 1 & $x^{6}+x^{5}-6 x^{4}-6 x^{3}+4 x^{2}+2 x-1$ \\
\hline-507328 & $-2^{6} \times 7927$ & S6 & 1 & $x^{6}+2 x^{5}-4 x^{4}-8 x^{3}+2 x^{2}+6 x+2$ \\
\hline-508075 & $-5^{2} \times 20323$ & S6 & 1 & $x^{6}-2 x^{4}-7 x^{3}+10 x+5$ \\
\hline-508480 & $-2^{6} \times 5 \times 7 \times 227$ & S6 & 1 & $x^{6}-7 x^{4}-2 x^{3}+6 x^{2}-4 x+1$ \\
\hline-508663 & $-223 \times 2281$ & S6 & 1 & $x^{6}+3 x^{5}-x^{4}-7 x^{3}-2 x^{2}+x+1$ \\
\hline-509451 & $-3 \times 169817$ & S6 & 1 & $x^{6}+x^{5}-7 x^{4}-10 x^{3}+6 x^{2}+11 x+1$ \\
\hline-512363 & $-17 \times 30139$ & S6 & 1 & $x^{6}-8 x^{4}-x^{3}+12 x^{2}-4 x-1$ \\
\hline
\end{tabular}




$\begin{array}{ll}-515308 & -2^{2} \times 47 \times 2741 \\ -516267 & -3^{3} \times 19121 \\ -516419 & -23 \times 22453 \\ -519051 & -3 \times 13 \times 13309 \\ -523519 & -523519 \\ -525995 & -5 \times 105199 \\ -526912 & -2^{6} \times 8233 \\ -526979 & -53 \times 61 \times 163 \\ -530923 & -241 \times 2203 \\ -533923 & -13 \times 67 \times 613 \\ -535027 & -571 \times 937 \\ -535543 & -29 \times 59 \times 313 \\ -535972 & -2^{2} \times 133993 \\ -546808 & -2^{3} \times 68351 \\ -554699 & -554699 \\ -556283 & -7 \times 13 \times 6113 \\ -561863 & -101 \times 5563 \\ -563011 & -563011 \\ -563911 & -643 \times 877 \\ -570539 & -570539 \\ -571803 & -3 \times 23 \times 8287 \\ -575408 & -2^{4} \times 35963 \\ -578671 & -73 \times 7927 \\ -579003 & -3 \times 17 \times 11353 \\ -580147 & -59 \times 9833 \\ -580687 & -580687 \\ -582464 & -2^{6} \times 19 \times 479 \\ -582611 & -61 \times 9551 \\ -582923 & -11 \times 197 \times 269 \\ -586731 & -3 \times 257 \times 761 \\ -593143 & -593143 \\ -595019 & -193 \times 3083 \\ -597591 & -3^{3} \times 22133 \\ -602695 & -5 \times 120539 \\ -612263 & -612263 \\ -612767 & -101 \times 6067 \\ -613131 & -3 \times 204377 \\ -6176999 & -3 \times 139 \times 1483 \\ -3632 & -306281 \\ -511 \times 883\end{array}$

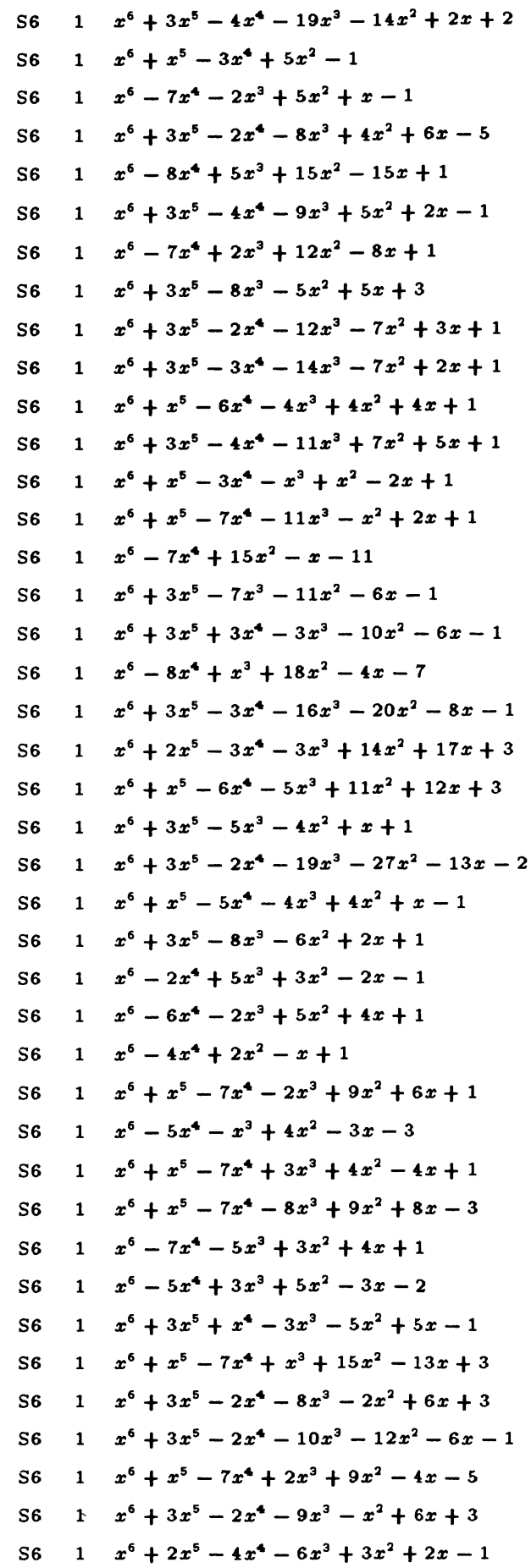




\begin{tabular}{|c|c|c|c|c|}
\hline-626011 & -626011 & S6 & 1 & $x^{6}+x^{5}-5 x^{4}-2 x^{3}+11 x^{2}+2 x-7$ \\
\hline-626531 & $-61 \times 10271$ & S6 & 1 & $x^{6}+x^{5}-2 x^{4}-x^{3}-x^{2}-2 x+1$ \\
\hline-628339 & $-31 \times 20269$ & S6 & 1 & $x^{6}+x^{5}-5 x^{4}+4 x^{2}-x-1$ \\
\hline-629952 & $-2^{6} \times 3 \times 17 \times 193$ & S6 & 1 & $x^{6}+2 x^{5}-3 x^{4}-6 x^{3}+2 x+1$ \\
\hline-635587 & $-467 \times 1361$ & S6 & 1 & $x^{6}+x^{5}-6 x^{4}-x^{3}+9 x^{2}-6 x+1$ \\
\hline-637547 & $-37 \times 17231$ & S6 & 1 & $x^{6}+2 x^{5}-5 x^{4}-15 x^{3}-8 x^{2}+3 x+1$ \\
\hline-638599 & $-13 \times 49123$ & S6 & 1 & $x^{6}+2 x^{5}-5 x^{4}-5 x^{3}+9 x^{2}-4 x+1$ \\
\hline-640027 & -640027 & S6 & 1 & $x^{6}-7 x^{4}-2 x^{3}+7 x^{2}+5 x+1$ \\
\hline-640176 & $-2^{4} \times 3 \times 13337$ & S6 & 1 & $x^{6}+3 x^{5}-4 x^{4}-19 x^{3}-16 x^{2}-3 x-1$ \\
\hline-644507 & -644507 & S6 & 1 & $x^{6}+x^{5}-7 x^{4}-2 x^{3}+11 x^{2}-6 x+1$ \\
\hline-645779 & $-17 \times 37987$ & S6 & 1 & $x^{6}+x^{5}-6 x^{4}-6 x^{3}+x^{2}+3 x+1$ \\
\hline-652459 & $-89 \times 7331$ & S6 & 1 & $x^{6}+x^{5}-7 x^{4}-4 x^{3}+12 x^{2}-3 x-1$ \\
\hline-653248 & $-2^{6} \times 59 \times 173$ & S6 & 1 & $x^{6}+2 x^{5}-6 x^{4}-8 x^{3}+9 x^{2}+6 x-5$ \\
\hline-658043 & -658043 & S6 & 1 & $x^{6}+x^{5}-6 x^{4}-2 x^{3}+8 x^{2}+2 x-3$ \\
\hline-659239 & $-7 \times 41 \times 2297$ & S6 & 1 & $x^{6}-7 x^{4}-3 x^{3}+9 x^{2}+6 x+1$ \\
\hline-661711 & $-619 \times 1069$ & S6 & 1 & $x^{6}-3 x^{4}-3 x^{3}+5 x^{2}+3 x-2$ \\
\hline-661831 & $-97 \times 6823$ & S6 & 1 & $x^{6}+x^{5}-5 x^{4}-3 x^{3}+6 x^{2}+3 x-2$ \\
\hline-662499 & $-3^{4} \times 8179$ & S6 & 1 & $x^{6}+x^{5}-7 x^{4}-6 x^{3}+13 x^{2}+8 x-1$ \\
\hline-662931 & $-3^{3} \times 43 \times 571$ & S6 & 1 & $x^{6}+3 x^{5}-2 x^{4}-4 x^{3}+7 x^{2}-5 x+1$ \\
\hline-663939 & $-3^{2} \times 73771$ & S6 & 1 & $x^{6}+x^{5}-2 x^{4}+2 x^{3}+2 x^{2}-2 x-1$ \\
\hline-664567 & $-53 \times 12539$ & S6 & 1 & $x^{6}+x^{5}-7 x^{4}+7 x^{3}+2 x^{2}-4 x+1$ \\
\hline-665323 & $-19^{3} \times 97$ & $\mathrm{~S} 6$ & 1 & $x^{6}+3 x^{5}-2 x^{4}-6 x^{3}-x^{2}+3 x+1$ \\
\hline-668347 & -668347 & S6 & 1 & $x^{6}+3 x^{5}-3 x^{4}-12 x^{3}+3 x^{2}+10 x+1$ \\
\hline-668783 & $-613 \times 1091$ & S6 & 1 & $x^{6}+x^{5}-7 x^{4}-2 x^{3}+7 x^{2}+x-2$ \\
\hline-669872 & $-2^{4} \times 7 \times 5981$ & s6 & 1 & $x^{6}+x^{5}-6 x^{4}-3 x^{3}+10 x^{2}-x-1$ \\
\hline-672003 & $-3^{3} \times 24889$ & S6 & 1 & $x^{6}+x^{5}-7 x^{4}-4 x^{3}+13 x^{2}-3$ \\
\hline .672379 & -672379 & S6 & 1 & $x^{6}+x^{5}-7 x^{4}-10 x^{3}+7 x^{2}+14 x+3$ \\
\hline-677783 & -677783 & S6 & 1 & $x^{6}+x^{5}-7 x^{4}-x^{3}+14 x^{2}-8 x+1$ \\
\hline-683083 & -683083 & S6 & 1 & $x^{6}+x^{5}-6 x^{4}-2 x^{3}+12 x^{2}-2 x-5$ \\
\hline-684043 & $-23 \times 29741$ & S6 & 1 & $x^{6}+3 x^{5}-2 x^{4}-5 x^{3}+7 x^{2}-2 x-1$ \\
\hline-688927 & $-691 \times 997$ & S6 & 1 & $x^{6}+3 x^{5}-x^{4}-9 x^{3}-5 x^{2}+3 x+1$ \\
\hline-691531 & -691531 & S6 & 1 & $x^{6}-7 x^{4}-4 x^{3}+3 x^{2}-3 x+1$ \\
\hline-695307 & $-3 \times 53 \times 4373$ & S6 & 1 & $x^{6}+3 x^{5}-2 x^{4}-10 x^{3}-2 x^{2}+6 x+1$ \\
\hline-697511 & -697511 & S6 & 1 & $x^{6}+x^{5}-3 x^{4}-5 x^{3}+x^{2}+5 x+1$ \\
\hline-698167 & $-809 \times 863$ & S6 & 1 & $x^{6}+3 x^{5}-4 x^{4}-7 x^{3}+x^{2}+5 x+2$ \\
\hline-700267 & $-307 \times 2281$ & S6 & 1 & $x^{6}+x^{5}-4 x^{4}-x^{3}+3 x^{2}-2 x+1$ \\
\hline-701387 & $-41 \times 17107$ & S6 & 1 & $x^{6}-8 x^{4}+4 x^{3}+8 x^{2}+x-1$ \\
\hline-702187 & $-73 \times 9619$ & s6 & 1 & $x^{6}+3 x^{5}-2 x^{4}-16 x^{3}-14 x^{2}+8 x+11$ \\
\hline-702691 & $-11 \times 127 \times 503$ & S6 & 1 & $x^{6}+x^{3}-6 x^{2}-2 x+5$ \\
\hline-703744 & $-2^{8} \times 2749$ & $\mathrm{~S} 6$ & 1 & $x^{6}-7 x^{4}-6 x^{3}+9 x^{2}+6 x-5$ \\
\hline-707479 & $-43 \times 16453$ & S6 & 1 & $x^{6}+3 x^{5}+x^{4}-5 x^{3}-6 x^{2}+1$ \\
\hline
\end{tabular}




\begin{tabular}{ll}
-709783 & $-19 \times 37357$ \\
-710251 & $-167 \times 4253$ \\
-710267 & $-67 \times 10601$ \\
-711219 & $-3 \times 237073$ \\
-712603 & -712603 \\
-713392 & $-2^{4} \times 44587$ \\
-715639 & -715639 \\
-717347 & $-23 \times 31189$ \\
-720007 & -720007 \\
-724167 & $-3^{3} \times 26821$ \\
-725803 & $-13 \times 31 \times 1801$ \\
-727744 & $-2^{6} \times 83 \times 137$ \\
-728512 & $-2^{6} \times 11383$ \\
-728887 & $-181 \times 4027$ \\
-729367 & -729367 \\
-732723 & $-3 \times 467 \times 523$ \\
-733588 & $-2^{2} \times 183397$ \\
-734483 & $-19 \times 29 \times 31 \times 43$ \\
-739399 & -739399 \\
-739663 & $-137 \times 5399$ \\
-740528 & $-2^{4} \times 31 \times 1493$ \\
-745907 & $-157 \times 4751$ \\
-746835 & $-3 \times 5 \times 49789$ \\
\hline
\end{tabular}

$\begin{array}{lll}\text { S6 } & 1 & x^{6}-4 x^{4}-8 x^{3}+15 x^{2}-7 x+1 \\ \text { S6 } & 1 & x^{6}+3 x^{5}-x^{4}-10 x^{3}-6 x^{2}+3 x+1 \\ \text { S6 } & 1 & x^{6}+3 x^{5}-2 x^{4}-9 x^{3}+x^{2}+8 x+1 \\ \text { S6 } & 1 & x^{6}+3 x^{5}-x^{4}-4 x^{3}+4 x^{2}+x-1 \\ \text { S6 } & 1 & x^{6}+3 x^{5}-2 x^{4}-11 x^{3}-5 x^{2}+2 x-1 \\ \text { S6 } & 1 & x^{6}+3 x^{5}-2 x^{4}-11 x^{3}-2 x^{2}+5 x-1 \\ \text { S6 } & 1 & x^{6}+3 x^{5}-2 x^{4}-9 x^{3}-x^{2}+7 x+2 \\ \text { S6 } & 1 & x^{6}+x^{5}-6 x^{4}-4 x^{3}+5 x^{2}+5 x+1 \\ \text { S6 } & 1 & x^{6}+x^{5}-7 x^{4}-5 x^{3}+5 x^{2}+5 x+1 \\ \text { S6 } & 1 & x^{6}+x^{5}-7 x^{4}-3 x^{3}+6 x^{2}+5 x+1 \\ \text { S6 } & 1 & x^{6}+x^{5}-7 x^{4}-14 x^{3}-13 x^{2}-6 x-1 \\ \text { S6 } & 1 & x^{6}+2 x^{5}-5 x^{4}-10 x^{3}+5 x^{2}+10 x-1 \\ \text { S6 } & 1 & x^{6}+2 x^{5}-3 x^{4}-12 x^{3}-17 x^{2}-10 x-2 \\ \text { S6 } & 1 & x^{6}-6 x^{4}-x^{3}+5 x^{2}+4 x+1 \\ \text { S6 } & 1 & x^{6}+x^{5}-7 x^{4}-11 x^{3}+x-4 \\ \text { S6 } & 1 & x^{6}+3 x^{5}-x^{4}-8 x^{3}-2 x^{2}+5 x-1 \\ \text { S6 } & 1 & x^{6}+x^{5}-3 x^{4}-5 x^{3}+3 x^{2}+4 x-2 \\ \text { S6 } & 1 & x^{6}+3 x^{5}-2 x^{4}-12 x^{3}-5 x^{2}+11 x+9 \\ \text { S6 } & 1 & x^{6}+x^{5}-6 x^{4}-9 x^{3}+3 x^{2}+7 x+2 \\ \text { S6 } & 1 & x^{6}+3 x^{5}-3 x^{4}-8 x^{3}+x^{2}+5 x+2 \\ \text { S6 } & 1 & x^{6}+x^{5}-6 x^{4}-7 x^{3}+8 x^{2}+11 x+1 \\ \text { S6 } & 1 & x^{6}+3 x^{5}-2 x^{4}-12 x^{3}-5 x^{2}+7 x+3 \\ \text { S6 } & 1 & x^{6}+3 x^{5}-3 x^{4}-12 x^{3}+2 x^{2}+13 x+1\end{array}$




\section{SIGNATURE $(2,2)$ PRIMITIF}

LISTE DES CORPS DE NOMBRES, DE SIGNATURE (2,2), DE DEGRÉ 6, DE DISCRIMINANT EN VALEUR ABSOLUE INFÉRIEUR OU ÉGAL Ȧ 161800 , PRIMITIFS.

Cette table contient :

0 corps de type $\mathrm{A} 5$

0 corps de type $\mathrm{A} 6$

0 corps de type $\mathrm{S} 5$

200 corps de type $\mathbf{S} 6$

Soit au total :

200 corps dans cette signature.

$\begin{array}{lllll}29077 & 29077 & \text { S6 } & 1 & x^{6}+2 x^{5}-x^{4}-x^{2}-x+1 \\ 29189 & 17^{2} \times 101 & \text { S6 } & 1 & x^{6}-4 x^{4}+4 x^{2}+x-1 \\ 31133 & 163 \times 191 & \text { S6 } & 1 & x^{6}+x^{5}+2 x^{4}-x^{3}-3 x^{2}+1 \\ 31709 & 37 \times 857 & \text { S6 } & 1 & x^{6}-6 x^{4}-4 x^{3}+4 x^{2}+3 x+1 \\ 37253 & 37253 & \text { S6 } & 1 & x^{6}+x^{5}-4 x^{4}-4 x^{3}+3 x^{2}+3 x+1 \\ 37568 & 2^{6} \times 587 & \text { S6 } & 1 & x^{6}+2 x^{5}+x^{4}-4 x^{3}-6 x^{2}-4 x-1 \\ 39269 & 107 \times 367 & \text { S6 } & 1 & x^{6}+2 x^{5}-2 x^{4}-5 x^{3}+2 x^{2}+4 x-1 \\ 40277 & 40277 & \text { S6 } & 1 & x^{6}+2 x^{5}-5 x^{4}-3 x^{3}+4 x^{2}+x-1 \\ 41069 & 7 \times 5867 & \text { S6 } & 1 & x^{6}-2 x^{4}+2 x^{2}-x-1 \\ 45301 & 89 \times 509 & \text { S6 } & 1 & x^{6}+x^{5}-x^{2}-x-1 \\ 46757 & 46757 & \text { S6 } & 1 & x^{6}+x^{5}+2 x^{3}-4 x^{2}+1 \\ 47149 & 47149 & \text { S6 } & 1 & x^{6}+3 x^{5}-x^{4}+2 x^{3}+2 x^{2}-x+1 \\ 47669 & 73 \times 653 & \text { S6 } & 1 & x^{6}+x^{5}+4 x^{2}+4 x+1\end{array}$




\begin{tabular}{|c|c|}
\hline 47933 & 47933 \\
\hline 49781 & $67 \times 743$ \\
\hline 50173 & $131 \times 383$ \\
\hline 51757 & $73 \times 709$ \\
\hline 51869 & 51869 \\
\hline 55376 & $2^{4} \times 3461$ \\
\hline 57152 & $2^{6} \times 19 \times 47$ \\
\hline 58589 & $41 \times 1429$ \\
\hline 58673 & $23 \times 2551$ \\
\hline 61193 & $11 \times 5563$ \\
\hline 62437 & $29 \times 2153$ \\
\hline 63461 & $17 \times 3733$ \\
\hline 63909 & $3^{5} \times 263$ \\
\hline 64829 & $241 \times 269$ \\
\hline 65405 & $5 \times 103 \times 127$ \\
\hline 65797 & $19 \times 3463$ \\
\hline 66413 & 66413 \\
\hline 67429 & 67429 \\
\hline 68389 & 68389 \\
\hline 70629 & $3 \times 13 \times 1811$ \\
\hline 70837 & $13 \times 5449$ \\
\hline 71021 & $29 \times 31 \times 79$ \\
\hline 74293 & 74293 \\
\hline 74517 & $3 \times 59 \times 421$ \\
\hline 74837 & $7 \times 10691$ \\
\hline 74941 & 74941 \\
\hline 75749 & $211 \times 359$ \\
\hline 76165 & $5 \times 15233$ \\
\hline 77461 & $71 \times 1091$ \\
\hline 77853 & $3 \times 25951$ \\
\hline 78301 & 78301 \\
\hline 78901 & 78901 \\
\hline 79029 & $3^{3} \times 2927$ \\
\hline 79517 & $131 \times 607$ \\
\hline 80021 & 80021 \\
\hline 82325 & $5^{2} \times 37 \times 89$ \\
\hline 82793 & 82793 \\
\hline 83477 & 83477 \\
\hline 84853 & $53 \times 1601$ \\
\hline 85133 & 85133 \\
\hline 85817 & 85817 \\
\hline
\end{tabular}

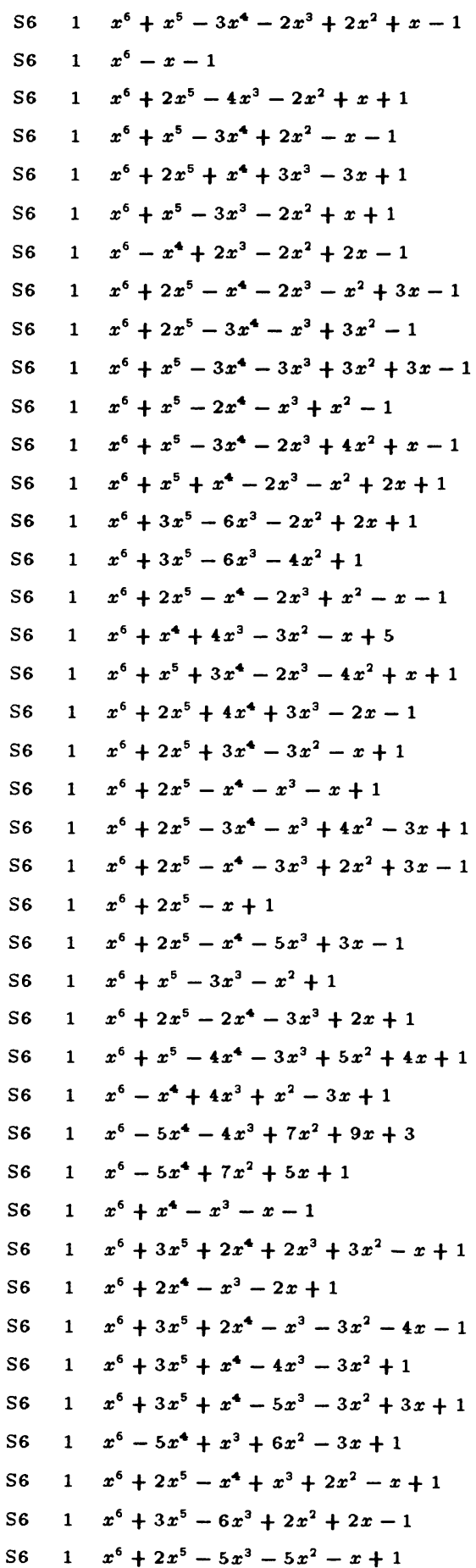




\begin{tabular}{|c|c|c|c|c|}
\hline 85952 & $2^{6} \times 17 \times 79$ & S6 & 1 & $x^{6}+2 x^{5}+2 x^{3}+x^{2}+1$ \\
\hline 86301 & $3^{2} \times 43 \times 223$ & S6 & 1 & $x^{6}+2 x^{5}+2 x^{4}-2 x^{2}-x-1$ \\
\hline 89501 & 89501 & S6 & 1 & $x^{6}+2 x^{4}+2 x^{3}-2 x^{2}-3 x-1$ \\
\hline 89653 & 89653 & S6 & 1 & $x^{6}+2 x^{5}-x^{4}-4 x^{3}-x^{2}+3 x+1$ \\
\hline 89737 & $19 \times 4723$ & S6 & 1 & $x^{6}+2 x^{5}+3 x^{3}+x^{2}+1$ \\
\hline 90137 & $23 \times 3919$ & S6 & 1 & $x^{6}+3 x^{5}-2 x^{4}-11 x^{3}-3 x^{2}+5 x-1$ \\
\hline 91093 & $71 \times 1283$ & S6 & 1 & $x^{6}+3 x^{5}+2 x^{4}+2 x^{2}+2 x-1$ \\
\hline 91597 & $11^{2} \times 757$ & 56 & 1 & $x^{6}+x^{5}+x^{2}-x-1$ \\
\hline 91769 & $163 \times 563$ & S6 & 1 & $x^{6}+2 x^{5}+x^{4}+8 x^{3}+18 x^{2}+11 x+2$ \\
\hline 92389 & $11 \times 37 \times 227$ & S6 & 1 & $x^{6}+2 x^{5}+x^{4}+2 x^{3}+x^{2}+x+1$ \\
\hline 92637 & $3^{3} \times 47 \times 73$ & S6 & 1 & $x^{6}+2 x^{5}-3 x^{4}-6 x^{3}+x^{2}+3 x-1$ \\
\hline 93037 & $7 \times 13291$ & S6 & 1 & $x^{6}+2 x^{4}-2 x^{3}+2 x^{2}-3 x+1$ \\
\hline 93253 & 93253 & S6 & 1 & $x^{6}-x^{4}-2 x^{3}+x^{2}+3 x+1$ \\
\hline 93629 & 93629 & S6 & 1 & $x^{6}+2 x^{5}-x^{4}-3 x^{3}-2 x^{2}-3 x-1$ \\
\hline 93904 & $2^{4} \times 5869$ & s6 & 1 & $x^{6}+x^{5}-x^{3}-x-1$ \\
\hline 94169 & 94169 & 56 & 1 & $x^{6}-6 x^{3}-x^{2}+3 x+1$ \\
\hline 94889 & 94889 & S6 & 1 & $x^{6}+x^{4}-x^{3}-2 x^{2}-2 x-1$ \\
\hline 95093 & 95093 & S6 & 1 & $x^{6}+5 x^{4}-4 x^{3}+7 x^{2}-5 x+1$ \\
\hline 95717 & 95717 & S6 & 1 & $x^{6}+2 x^{5}-2 x^{3}-2 x^{2}-x-1$ \\
\hline 95808 & $2^{6} \times 3 \times 499$ & S6 & 1 & $x^{6}+2 x^{5}-2 x^{4}-2 x^{3}+x^{2}+2 x-1$ \\
\hline 95852 & $2^{2} \times 31 \times 773$ & S6 & 1 & $x^{6}+x^{5}-4 x^{3}+3 x+1$ \\
\hline 95869 & 95869 & S6 & 1 & $x^{6}+x^{5}-4 x^{4}-3 x^{3}+5 x^{2}+1$ \\
\hline 96137 & 96137 & s6 & 1 & $x^{6}+2 x^{5}-3 x^{4}-4 x^{3}+6 x^{2}+3 x-4$ \\
\hline 96172 & $2^{2} \times 24043$ & S6 & 1 & $x^{6}+x^{5}+2 x^{4}-x^{2}-2 x-2$ \\
\hline 97197 & $3 \times 179 \times 181$ & S6 & 1 & $x^{6}+x^{5}-x^{3}-x^{2}+2 x-1$ \\
\hline 97697 & $151 \times 647$ & S6 & 1 & $x^{6}+2 x^{5}-x^{4}-3 x^{3}+x^{2}+x-2$ \\
\hline 97909 & $7 \times 71 \times 197$ & S6 & 1 & $x^{6}+3 x^{5}+2 x^{4}-2 x^{3}-4 x^{2}+4 x-1$ \\
\hline 97981 & $13 \times 7537$ & S6 & 1 & $x^{6}+2 x^{5}-x^{4}-3 x^{3}+3 x-1$ \\
\hline 98552 & $2^{3} \times 97 \times 127$ & S6 & 1 & $x^{6}+x^{5}+x^{4}+x^{3}-3 x^{2}-2 x+2$ \\
\hline 98993 & 98993 & S6 & 1 & $x^{6}+2 x^{5}-x^{3}-x^{2}-x-1$ \\
\hline 99136 & $2^{6} \times 1549$ & S6 & 1 & $x^{6}+2 x^{5}-2 x^{3}-3 x^{2}-2 x+1$ \\
\hline 100109 & 100109 & s6 & 1 & $x^{6}+2 x^{5}-2 x^{4}-5 x^{3}+2 x^{2}+4 x+1$ \\
\hline 100973 & $37 \times 2729$ & S6 & 1 & $x^{6}+3 x^{5}+x^{4}-6 x^{3}-7 x^{2}+2 x+5$ \\
\hline 101072 & $2^{4} \times 6317$ & S6 & 1 & $x^{6}+3 x^{5}-2 x^{4}-13 x^{3}-6 x^{2}+13 x+11$ \\
\hline 101441 & $19^{2} \times 281$ & S6 & 1 & $x^{6}+2 x^{5}+x^{4}+4 x^{3}+2 x^{2}+x+2$ \\
\hline 101653 & 101653 & S6 & 1 & $x^{6}+x^{5}+x^{2}+x-1$ \\
\hline 102693 & $3 \times 34231$ & S6 & 1 & $x^{6}+2 x^{5}+3 x^{4}+4 x^{3}+x^{2}+x-1$ \\
\hline 104173 & 104173 & s6 & 1 & $x^{6}+3 x^{5}-6 x^{3}+x^{2}+3 x-1$ \\
\hline 104453 & $67 \times 1559$ & s6 & 1 & $x^{6}+2 x^{5}+x^{3}+4 x^{2}+2 x+1$ \\
\hline 104717 & 104717 & S6 & 1 & $x^{6}-6 x^{4}+7 x^{3}+2 x^{2}-4 x+1$ \\
\hline 104872 & $2^{3} \times 13109$ & S6 & 1 & $x^{6}+2 x^{5}-x^{4}-x^{3}+x^{2}-x+1$ \\
\hline
\end{tabular}




\begin{tabular}{|c|c|}
\hline 105408 & $2^{6} \times 3^{3} \times 61$ \\
\hline 105601 & 105601 \\
\hline 105881 & $113 \times 937$ \\
\hline 106189 & 106189 \\
\hline 106229 & $19 \times 5591$ \\
\hline 106412 & $2^{2} \times 37 \times 719$ \\
\hline 106432 & $2^{6} \times 1663$ \\
\hline 106909 & $11 \times 9719$ \\
\hline 107417 & $163 \times 659$ \\
\hline 108829 & $7^{2} \times 2221$ \\
\hline 109325 & $5^{2} \times 4373$ \\
\hline 109673 & 109673 \\
\hline 110261 & 110261 \\
\hline 111152 & $2^{4} \times 6947$ \\
\hline 111269 & 111269 \\
\hline 111269 & 111269 \\
\hline 111661 & $11 \times 10151$ \\
\hline 112573 & 112573 \\
\hline 113728 & $2^{6} \times 1777$ \\
\hline 114069 & $3 \times 47 \times 809$ \\
\hline 114377 & 114377 \\
\hline 116048 & $2^{4} \times 7253$ \\
\hline 116053 & $7 \times 59 \times 281$ \\
\hline 116197 & $131 \times 887$ \\
\hline 116204 & $2^{2} \times 11 \times 19 \times 139$ \\
\hline 116701 & $13 \times 47 \times 191$ \\
\hline 116829 & $3^{3} \times 4327$ \\
\hline 116944 & $2^{4} \times 7309$ \\
\hline 117813 & $3 \times 173 \times 227$ \\
\hline 119104 & $2^{6} \times 1861$ \\
\hline 119797 & 119797 \\
\hline 121084 & $2^{2} \times 30271$ \\
\hline 122560 & $2^{6} \times 5 \times 383$ \\
\hline 122708 & $2^{2} \times 30677$ \\
\hline 122753 & 122753 \\
\hline 123344 & $2^{4} \times 13 \times 593$ \\
\hline 123377 & 123377 \\
\hline 124037 & $71 \times 1747$ \\
\hline 124333 & $11 \times 89 \times 127$ \\
\hline 124457 & $17 \times 7321$ \\
\hline 125201 & 125201 \\
\hline
\end{tabular}

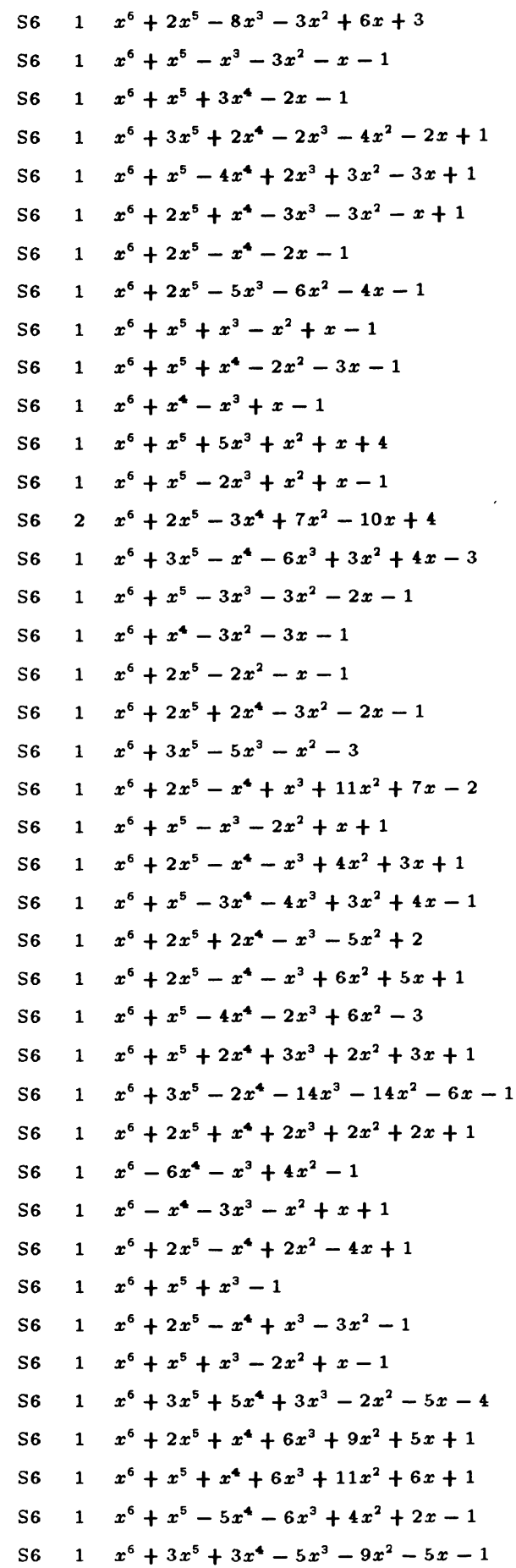




\begin{tabular}{|c|c|c|c|c|}
\hline 125297 & $107 \times 1171$ & S6 & 1 & $x^{6}+3 x^{5}+3 x^{4}-2 x^{2}-2 x+1$ \\
\hline 125332 & $2^{2} \times 31333$ & S6 & 1 & $x^{6}+3 x^{5}+4 x^{4}-3 x^{3}-6 x^{2}+2 x+1$ \\
\hline 125341 & $17 \times 73 \times 101$ & S6 & 1 & $x^{6}+2 x^{5}+2 x^{4}-2 x^{3}-2 x^{2}-x+1$ \\
\hline 126272 & $2^{6} \times 1973$ & S6 & 1 & $x^{6}+x^{4}-x^{2}-2 x-1$ \\
\hline 126453 & $3 \times 61 \times 691$ & S6 & 1 & $x^{6}+2 x^{5}-x^{4}-4 x^{3}+3 x^{2}+x-1$ \\
\hline 126493 & 126493 & S6 & 1 & $x^{6}+2 x^{5}-5 x^{3}+2 x^{2}+2 x-1$ \\
\hline 126517 & 126517 & S6 & 1 & $x^{6}+2 x^{5}-x^{4}-9 x^{3}-12 x^{2}-7 x-1$ \\
\hline 127168 & $2^{6} \times 1987$ & S6 & 1 & $x^{6}+2 x^{5}-x^{4}-4 x^{3}-2 x^{2}+1$ \\
\hline 128377 & 128377 & S6 & 1 & $x^{6}+2 x^{5}-x^{4}-4 x^{3}+2 x^{2}+3 x-2$ \\
\hline 128533 & $79 \times 1627$ & S6 & 1 & $x^{6}+x^{5}-2 x^{2}-2 x-1$ \\
\hline 129001 & 129001 & S6 & 1 & $x^{6}+2 x^{5}+x^{4}+x^{3}-1$ \\
\hline 129569 & $11 \times 11779$ & S6 & 1 & $x^{6}-2 x^{3}-x^{2}-x+1$ \\
\hline 129728 & $2^{6} \times 2027$ & s6 & 1 & $x^{6}+2 x^{5}-2 x^{3}-x^{2}+2 x+1$ \\
\hline 131849 & 131849 & S6 & 1 & $x^{6}+2 x^{5}+x^{3}+x^{2}-2 x+1$ \\
\hline 132032 & $2^{6} \times 2063$ & S6 & 1 & $x^{6}+2 x^{5}+4 x^{4}+2 x^{3}-x^{2}-2 x-1$ \\
\hline 132416 & $2^{6} \times 2069$ & S6 & 1 & $x^{6}+2 x^{5}+x^{4}-6 x^{3}-3 x^{2}+2 x+1$ \\
\hline 132661 & 132661 & S6 & 1 & $x^{6}+2 x^{5}-x^{4}+3 x^{2}-x+1$ \\
\hline 133553 & $7 \times 19079$ & S6 & 1 & $x^{6}+x^{5}+x^{4}-x^{3}+2 x^{2}-3 x+1$ \\
\hline 134012 & $2^{2} \times 33503$ & S6 & 1 & $x^{6}+x^{5}+4 x^{4}+6 x^{3}+8 x^{2}+5 x+1$ \\
\hline 134201 & $67 \times 2003$ & S6 & 1 & $x^{6}+2 x^{5}+x^{4}-4 x^{3}-8 x^{2}-7 x-2$ \\
\hline 135001 & $127 \times 1063$ & S6 & 1 & $x^{6}+2 x^{5}+2 x^{4}+x^{3}+x^{2}-x-1$ \\
\hline 136933 & $19 \times 7207$ & S6 & 1 & $x^{6}+2 x^{4}+2 x^{3}+3 x-1$ \\
\hline 137349 & $3^{3} \times 5087$ & S6 & 1 & $x^{6}+2 x^{5}+2 x^{4}+x^{3}-2 x^{2}-2 x+1$ \\
\hline 138693 & $3 \times 83 \times 557$ & S6 & 1 & $x^{6}+x^{5}-4 x^{3}-2 x^{2}+3$ \\
\hline 139453 & $37 \times 3769$ & S6 & 1 & $x^{6}+x^{5}-x^{2}+x-1$ \\
\hline 139777 & $11 \times 97 \times 131$ & S6 & 1 & $x^{6}+3 x^{5}-7 x^{3}-11 x^{2}-7 x-2$ \\
\hline 140697 & $3^{6} \times 193$ & S6 & 1 & $x^{6}-2 x^{3}-3 x^{2}-3 x-1$ \\
\hline 140861 & $7 \times 20123$ & S6 & 1 & $x^{6}+2 x^{5}+x^{4}+x^{3}+2 x^{2}+x-1$ \\
\hline 143773 & $7 \times 19 \times 23 \times 47$ & S6 & 1 & $x^{6}+2 x^{5}+x^{4}+2 x^{3}+3 x^{2}+3 x+1$ \\
\hline 145357 & $137 \times 1061$ & S6 & 1 & $x^{6}+3 x^{5}+4 x^{4}-5 x^{3}-3 x^{2}+4 x-1$ \\
\hline 147013 & $113 \times 1301$ & S6 & 1 & $x^{6}+2 x^{5}-x^{4}-x^{3}+2 x^{2}+x-1$ \\
\hline 147913 & $23 \times 59 \times 109$ & S6 & 1 & $x^{6}+3 x^{5}+2 x^{4}+x^{3}-x^{2}-x-1$ \\
\hline 147933 & $3^{3} \times 5479$ & S6 & 1 & $x^{6}+x^{5}+x^{3}-x^{2}+1$ \\
\hline 148573 & 148573 & S6 & 1 & $x^{6}+2 x^{5}-x^{4}-4 x^{3}-3 x^{2}-x+1$ \\
\hline 148581 & - $3^{3} \times 5503$ & S6 & 1 & $x^{6}+x^{5}+2 x^{2}-1$ \\
\hline 148605 & $3 \times 5 \times 9907$ & S6 & 1 & $x^{6}+3 x^{5}+2 x^{4}-5 x^{3}-7 x^{2}+5$ \\
\hline 148861 & 148861 & S6 & 1 & $x^{6}+2 x^{5}+3 x^{4}-3 x^{2}-5 x-3$ \\
\hline 148889 & $13^{2} \times 881$ & s6 & 1 & $x^{6}+2 x^{5}+x^{4}-x^{3}-x^{2}-2 x-1$ \\
\hline 149101 & 149101 & S6 & 1 & $x^{6}+2 x^{5}+2 x^{2}-x-1$ \\
\hline 149353 & $233 \times 641$ & $\mathrm{~S} 6$ & 1 & $x^{6}-x^{3}-x^{2}-x+1$ \\
\hline 149849 & $7 \times 21407$ & S6 & 1 & $x^{6}+2 x^{5}-x^{4}+4 x^{2}-3 x-4$ \\
\hline
\end{tabular}




$\begin{array}{ll}149925 & 3 \times 5^{2} \times 1999 \\ 150224 & 2^{4} \times 41 \times 229 \\ 150721 & 150721 \\ 151253 & 151253 \\ 151773 & 3 \times 50591 \\ 152177 & 43 \times 3539 \\ 152297 & 152297 \\ 152837 & 152837 \\ 153269 & 153269 \\ 153461 & 7 \times 11 \times 1993 \\ 154221 & 3 \times 51407 \\ 154361 & 163 \times 947 \\ 154456 & 2^{3} \times 43 \times 449 \\ 154477 & 179 \times 863 \\ 154689 & 3 \times 51563 \\ 155609 & 155609 \\ 155713 & 31 \times 5023 \\ 158381 & 251 \times 631 \\ 158461 & 211 \times 751 \\ 159037 & 359 \times 443 \\ 160421 & 59 \times 2719 \\ 160661 & 347 \times 463 \\ 161417 & 31 \times 41 \times 127\end{array}$

$$
\begin{array}{lll}
\text { S6 } & 1 & x^{6}+3 x^{5}+x^{4}-4 x^{3}-5 x^{2}+5 \\
\text { S6 } & 1 & x^{6}+x^{5}-x^{3}-2 x^{2}-x+1 \\
\text { S6 } & 1 & x^{6}+2 x^{5}-x^{4}-x^{3}+2 x^{2}+1 \\
\text { S6 } & 1 & x^{6}+2 x^{5}-3 x^{4}-12 x^{3}-9 x^{2}+3 x+5 \\
\text { S6 } & 1 & x^{6}+3 x^{4}+x^{3}+2 x^{2}+x-1 \\
\text { S6 } & 1 & x^{6}+2 x^{5}-x^{4}-3 x^{3}-4 x^{2}-2 x-1 \\
\text { S6 } & 1 & x^{6}+2 x^{5}-x^{4}-x^{3}+x^{2}-1 \\
\text { S6 } & 1 & x^{6}+2 x^{5}-x^{4}-2 x^{3}+3 x^{2}+x-1 \\
\text { S6 } & 1 & x^{6}+x^{5}-2 x-1 \\
\text { S6 } & 1 & x^{6}+2 x^{5}-4 x^{3}-6 x^{2}-3 x-1 \\
\text { S6 } & 1 & x^{6}+4 x^{4}+6 x^{3}+8 x^{2}+5 x+1 \\
\text { S6 } & 1 & x^{6}+x^{5}-5 x^{4}-3 x^{3}+9 x^{2}+3 x-5 \\
\text { S6 } & 1 & x^{6}+x^{5}-2 x^{3}-2 x^{2}-x+1 \\
\text { S6 } & 1 & x^{6}+x^{5}-2 x^{4}+2 x-1 \\
\text { S6 } & 1 & x^{6}+2 x^{5}+4 x^{4}+5 x^{3}+3 x^{2}+3 x-1 \\
\text { S6 } & 1 & x^{6}+x^{5}+3 x^{3}+x^{2}+x+1 \\
\text { S6 } & 1 & x^{6}+x^{5}+x^{4}+x^{3}-2 x^{2}-1 \\
\text { S6 } & 1 & x^{6}+2 x^{5}-4 x^{3}-8 x^{2}-5 x-1 \\
\text { S6 } & 1 & x^{6}+2 x^{5}+x^{4}+5 x^{3}+4 x^{2}-5 x+1 \\
\text { S6 } & 1 & x^{6}+x^{5}-x^{2}+x+1 \\
\text { S6 } & 1 & x^{6}+2 x^{5}-2 x^{3}-2 x^{2}+x+3 \\
\text { S6 } & 1 & x^{6}+3 x^{5}-7 x^{3}+3 x^{2}+2 x-1 \\
\text { S6 } & 1 & x^{6}-3 x^{4}+4 x^{2}-x-2 \\
& &
\end{array}
$$




\section{Signature $(0,3)$ PRIMItif}

LISTE DES CORPS DE NOMBRES, DE SIGNATURE (0,3), DE DEGRÉ 6, DE DISCRIMINANT EN VALEUR ABSOLUE INFÉRIEUR OU ÉGAL À 191000,

\section{PRIMITIFS.}

Cette table contient :

0 corps de type A5

0 corps de type A6

0 corps de type S5

200 corps de type $\mathrm{S} 6$

Soit au total :

200 corps dans cette signature.

$\begin{array}{ll}-14731 & -14731 \\ -20627 & -20627 \\ -22291 & -22291 \\ -25747 & -25747 \\ -27971 & -83 \times 337 \\ -30808 & -2^{3} \times 3851 \\ -31223 & -31223 \\ -32171 & -53 \times 607 \\ -32911 & -32911 \\ -33791 & -33791 \\ -35099 & -35099 \\ -36107 & -36107 \\ -36235 & -5 \times 7247\end{array}$

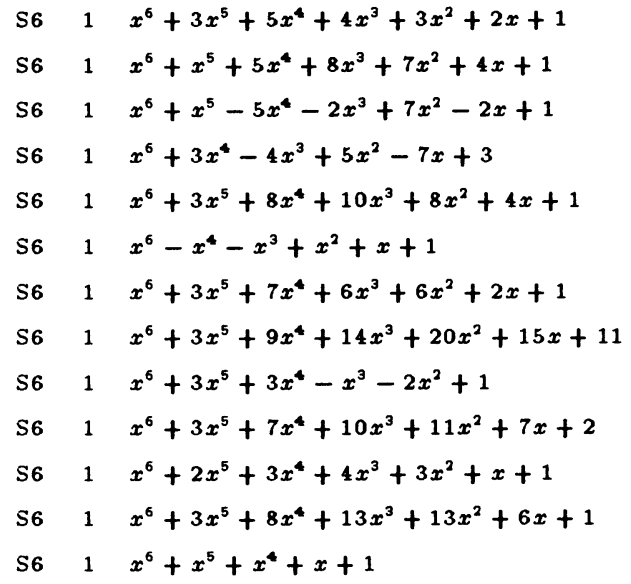




\begin{tabular}{|c|c|}
\hline-37423 & -37423 \\
\hline-37463 & -37463 \\
\hline-40543 & -40543 \\
\hline-42316 & $-2^{2} \times 71 \times 149$ \\
\hline-43063 & -43063 \\
\hline-43531 & $-101 \times 431$ \\
\hline-44423 & $-31 \times 1433$ \\
\hline-45203 & $-17 \times 2659$ \\
\hline-45223 & $-41 \times 1103$ \\
\hline-45508 & $-2^{2} \times 31 \times 367$ \\
\hline-45872 & $-2^{4} \times 47 \times 61$ \\
\hline-46651 & $-11 \times 4241$ \\
\hline-47552 & $-2^{6} \times 743$ \\
\hline-49615 & $-5 \times 9923$ \\
\hline-50587 & -50587 \\
\hline-52271 & $-167 \times 313$ \\
\hline-52891 & $-227 \times 233$ \\
\hline-54691 & $-7 \times 13 \times 601$ \\
\hline-59555 & $-5 \times 43 \times 277$ \\
\hline-60175 & $-5^{2} \times 29 \times 83$ \\
\hline-60556 & $-2^{2} \times 15139$ \\
\hline-60719 & -60719 \\
\hline-61131 & $-3 \times 7 \times 41 \times 71$ \\
\hline-62299 & -62299 \\
\hline-63023 & $-19 \times 31 \times 107$ \\
\hline-63071 & $-59 \times 1069$ \\
\hline-63436 & $-2^{2} \times 15859$ \\
\hline-65231 & $-37 \times 41 \times 43$ \\
\hline-65991 & $-3 \times 21997$ \\
\hline-66679 & $-131 \times 509$ \\
\hline-66928 & $-2^{4} \times 47 \times 89$ \\
\hline-67595 & $-5 \times 11 \times 1229$ \\
\hline-68587 & $-107 \times 641$ \\
\hline-70888 & $-2^{3} \times 8861$ \\
\hline-70967 & $-13 \times 53 \times 103$ \\
\hline-72063 & $-3^{3} \times 17 \times 157$ \\
\hline-73228 & $-2^{2} \times 18307$ \\
\hline-75559 & $-11 \times 6869$ \\
\hline-77507 & $-179 \times 433$ \\
\hline-78947 & $-11 \times 7177$ \\
\hline-80192 & $-2^{6} \times 7 \times 179$ \\
\hline
\end{tabular}

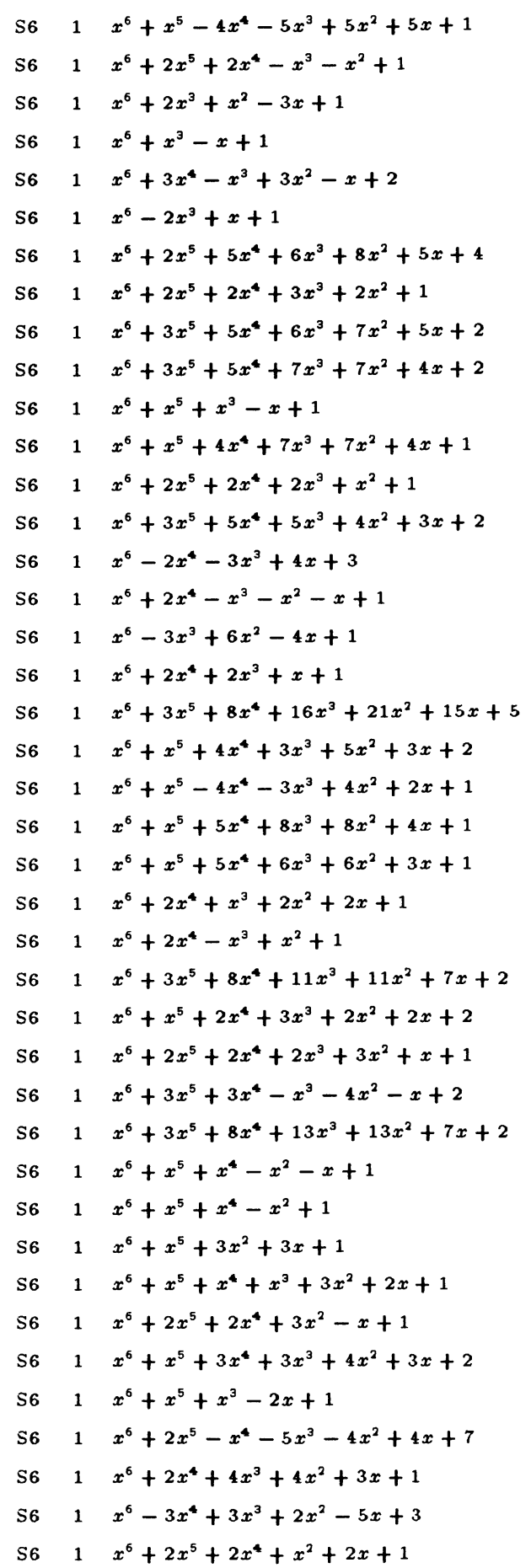




\begin{tabular}{|c|c|c|c|c|}
\hline-81416 & $-2^{3} \times 10177$ & S6 & 1 & $x^{6}+2 x^{5}-3 x^{3}-x^{2}+2 x+2$ \\
\hline-81608 & $-2^{3} \times 101^{2}$ & S6 & 1 & $x^{6}+3 x^{5}+x^{4}+x^{3}+5 x^{2}-2 x+2$ \\
\hline-82343 & $-67 \times 1229$ & $\mathrm{~s} 6$ & 1 & $x^{6}+2 x^{4}+3 x^{2}-x+1$ \\
\hline-82559 & -82559 & S6 & 1 & $x^{6}+3 x^{5}+5 x^{4}+7 x^{3}+7 x^{2}+5 x+3$ \\
\hline-83023 & -83023 & S6 & 1 & $x^{6}+2 x^{5}+2 x^{4}+x^{2}+x+1$ \\
\hline-83667 & $-3 \times 167 \times 167$ & S6 & 1 & $x^{6}+2 x^{2}+x+1$ \\
\hline-86111 & -86111 & S6 & 1 & $x^{6}+x^{5}+x^{4}-x^{3}+2 x+1$ \\
\hline-86171 & -86171 & S6 & 1 & $x^{6}+2 x^{4}+3 x^{3}+4 x^{2}+2 x+1$ \\
\hline-87767 & -87767 & S6 & 1 & $x^{6}-x^{4}-x^{3}+x^{2}+1$ \\
\hline-89363 & -89363 & S6 & 1 & $x^{6}+x^{5}+2 x^{4}-2 x^{3}+x^{2}-2 x+1$ \\
\hline-89627 & -89627 & S6 & 1 & $x^{6}+4 x^{4}+4 x^{2}-x+1$ \\
\hline-90047 & $-53 \times 1699$ & S6 & 1 & $x^{6}+4 x^{4}-x^{3}+5 x^{2}-x+1$ \\
\hline-92051 & -92051 & S6 & 1 & $x^{6}+x^{5}+5 x^{4}+4 x^{3}+6 x^{2}+3 x+1$ \\
\hline-93895 & $-5 \times 89 \times 211$ & S6 & 1 & $x^{6}+2 x^{4}-x^{3}+3 x^{2}-2 x+1$ \\
\hline-94111 & -94111 & S6 & 1 & $x^{6}+2 x^{4}-2 x^{3}+3 x^{2}-3 x+1$ \\
\hline-94475 & $-5^{2} \times 3779$ & S6 & 1 & $x^{6}+2 x^{5}+2 x^{4}+3 x^{3}+4 x^{2}+2 x+1$ \\
\hline-95667 & $-3 \times 11 \times 13 \times 223$ & S6 & 1 & $x^{6}+x^{5}+x^{4}-x+1$ \\
\hline-98096 & $-2^{4} \times 6131$ & S6 & 1 & $x^{6}+x^{5}+x^{3}+2 x^{2}+x+1$ \\
\hline-98591 & $-19 \times 5189$ & S6 & 1 & $x^{6}+2 x^{4}+x^{2}-x+1$ \\
\hline .99248 & $-2^{4} \times 6203$ & S6 & 1 & $x^{6}+3 x^{5}-2 x^{3}+2 x^{2}-4 x+2$ \\
\hline-101368 & $-2^{3} \times 12671$ & S6 & 1 & $x^{6}+3 x^{5}+x^{4}-3 x^{3}-x^{2}+1$ \\
\hline-101975 & $-5^{2} \times 4079$ & S6 & 1 & $x^{6}+3 x^{5}+4 x^{4}+3 x^{3}+7 x^{2}+5 x+1$ \\
\hline-104128 & $-2^{6} \times 1627$ & S6 & 1 & $x^{6}+3 x^{5}+x^{4}-2 x^{3}+3 x^{2}+9 x+7$ \\
\hline-104551 & -104551 & S6 & 1 & $x^{6}+2 x^{4}-2 x^{3}+3 x^{2}-x+1$ \\
\hline-109735 & $-5 \times 17 \times 1291$ & S6 & 1 & $x^{6}+2 x^{5}+2 x^{4}-x^{3}-x^{2}+x+1$ \\
\hline-110499 & $-3 \times 36833$ & S6 & 1 & $x^{6}+x^{5}+4 x^{4}+3 x^{3}+3 x^{2}+2 x+1$ \\
\hline-110863 & -110863 & S6 & 1 & $x^{6}+x^{5}+x^{4}-2 x^{3}+4 x^{2}-2 x+1$ \\
\hline-111191 & -111191 & s6 & 1 & $x^{6}-3 x^{3}-2 x^{2}+3 x+2$ \\
\hline-111236 & $-2^{2} \times 27809$ & S6 & 1 & $x^{6}+x^{5}+3 x^{4}+7 x^{3}+11 x^{2}+8 x+2$ \\
\hline-111743 & $-131 \times 853$ & S6 & 1 & $x^{6}+4 x^{4}-x^{3}+5 x^{2}-2 x+1$ \\
\hline-112748 & $-2^{2} \times 71 \times 397$ & S6 & 1 & $x^{6}+3 x^{5}+8 x^{4}+13 x^{3}+14 x^{2}+8 x+2$ \\
\hline-113132 & $-2^{2} \times 28283$ & S6 & 1 & $x^{6}+3 x^{5}+8 x^{4}+11 x^{3}+12 x^{2}+8 x+3$ \\
\hline-113471 & $-233 \times 487$ & S6 & 1 & $x^{6}+2 x^{5}+2 x^{4}+x^{3}-x^{2}-x+1$ \\
\hline-114443 & $-7 \times 16349$ & S6 & 1 & $x^{6}+2 x^{5}+2 x^{4}+2 x^{2}+3 x+1$ \\
\hline-115279 & -115279 & S6 & 1 & $x^{6}+3 x^{5}+7 x^{4}+10 x^{3}+9 x^{2}+5 x+2$ \\
\hline-118476 & $-2^{2} \times 3^{3} \times 1097$ & S6 & 1 & $x^{6}+x^{5}+x^{3}+2 x^{2}+1$ \\
\hline-119095 & $-5 \times 23819$ & 56 & 1 & $x^{6}+x^{5}+x^{4}+3 x^{3}+4 x^{2}-x+5$ \\
\hline-120863 & -120863 & $\mathrm{~s} 6$ & 1 & $x^{6}+2 x^{4}-x^{3}+x^{2}+3 x+1$ \\
\hline-121115 & $-5 \times 24223$ & 56 & 1 & $x^{6}+3 x^{5}+5 x^{4}+4 x^{3}+x^{2}+1$ \\
\hline-122347 & -122347 & S6 & 1 & $x^{6}+2 x^{5}+3 x^{4}-2 x^{2}-x+1$ \\
\hline-122708 & $-2^{2} \times 30677$ & $\mathrm{~S} 6$ & 1 & $x^{6}+x^{5}+3 x^{4}-x^{3}-x^{2}+2 x+1$ \\
\hline
\end{tabular}




\begin{tabular}{|c|c|c|c|c|}
\hline-122711 & $-277 \times 143$ & S6 & 1 & $x^{6}+x^{5}+3 x^{4}-x^{3}+3 x^{2}-x+1$ \\
\hline-123072 & $-2^{6} \times 3 \times 641$ & S6 & 1 & $x^{6}+2 x^{5}+2 x^{4}+2 x^{3}+5 x^{2}+6 x+3$ \\
\hline-123791 & -123791 & S6 & 1 & $x^{6}+x^{5}+4 x^{4}+3 x^{3}+7 x^{2}+3 x+4$ \\
\hline-124136 & $-2^{3} \times 59 \times 263$ & S6 & 1 & $x^{6}+2 x^{5}+7 x^{4}+9 x^{3}+13 x^{2}+9 x+5$ \\
\hline-125031 & $-3 \times 71 \times 587$ & S6 & 1 & $x^{6}+2 x^{5}+3 x^{4}+x^{3}+x^{2}+x+2$ \\
\hline-125075 & $-5^{2} \times 5003$ & S6 & 1 & $x^{6}+x^{5}+4 x^{4}+2 x^{3}+4 x^{2}+2 x+1$ \\
\hline-125488 & $-2^{4} \times 11 \times 23 \times 31$ & S6 & 1 & $x^{6}+x^{5}+4 x^{4}+4 x^{3}+6 x^{2}+4 x+2$ \\
\hline-125591 & -125591 & S6 & 1 & $x^{6}+5 x^{4}-x^{3}+8 x^{2}-2 x+5$ \\
\hline-125744 & $-2^{4} \times 29 \times 271$ & S6 & 1 & $x^{6}+3 x^{5}+2 x^{4}-3 x^{3}-2 x^{2}+x+1$ \\
\hline-127079 & -127079 & S6 & 1 & $x^{6}+3 x^{5}+7 x^{4}+7 x^{3}+5 x^{2}+3 x+1$ \\
\hline-127359 & $-3^{3} \times 53 \times 89$ & S6 & 1 & $x^{6}+3 x^{5}+5 x^{4}+11 x^{3}+15 x^{2}+9 x+3$ \\
\hline-128747 & -128747 & S6 & 1 & $x^{6}+3 x^{5}+8 x^{4}+9 x^{3}+9 x^{2}+2 x+1$ \\
\hline-129328 & $-2^{4} \times 59 \times 137$ & S6 & 1 & $x^{6}+x^{5}+x^{3}+4 x^{2}+3 x+1$ \\
\hline-129647 & $-7 \times 18521$ & S6 & 1 & $x^{6}+3 x^{5}+5 x^{4}+9 x^{3}+9 x^{2}+7 x+7$ \\
\hline-130471 & $-11 \times 29 \times 409$ & S6 & 1 & $x^{6}+4 x^{4}-x^{3}+3 x^{2}-x+1$ \\
\hline .130911 & $-3 \times 11 \times 3967$ & S6 & 1 & $x^{6}+4 x^{4}+3 x^{3}+5 x^{2}+2 x+1$ \\
\hline-131463 & $-3^{5} \times 541$ & S6 & 1 & $x^{6}+x^{5}+x^{4}+2 x^{3}+6 x^{2}+6 x+3$ \\
\hline-131863 & $-29 \times 4547$ & S6 & 1 & $x^{6}+2 x^{5}+2 x^{4}+3 x^{3}+2 x^{2}+x+2$ \\
\hline-132555 & $-3 \times 5 \times 8837$ & S6 & 1 & $x^{6}+3 x^{5}+7 x^{4}+12 x^{3}+14 x^{2}+9 x+3$ \\
\hline-132996 & $-2^{2} \times 3 \times 11083$ & S6 & 1 & $x^{6}+3 x^{5}+8 x^{4}+12 x^{3}+14 x^{2}+9 x+3$ \\
\hline-133067 & $-11 \times 12097$ & S6 & 1 & $x^{6}+x^{5}+x^{3}+3 x^{2}+2 x+1$ \\
\hline-133351 & -133351 & S6 & 1 & $x^{6}+2 x^{4}-x^{3}+3 x^{2}+1$ \\
\hline-134363 & -134363 & S6 & 1 & $x^{6}+2 x^{5}+2 x^{4}+x+2$ \\
\hline-134779 & $-53 \times 2543$ & S6 & 1 & $x^{6}+x^{5}+4 x^{4}+x^{3}+3 x^{2}-2 x+1$ \\
\hline-136607 & -136607 & 56 & 1 & $x^{6}+5 x^{4}-x^{3}+7 x^{2}-2 x+3$ \\
\hline-138296 & $-2^{3} \times 59 \times 293$ & s6 & 1 & $x^{6}+3 x^{5}+7 x^{4}+11 x^{3}+11 x^{2}+6 x+2$ \\
\hline-139507 & $-61 \times 2287$ & S6 & 1 & $x^{6}+3 x^{5}+9 x^{4}+12 x^{3}+9 x^{2}+4 x+1$ \\
\hline-141248 & $-2^{6} \times 2207$ & S6 & 1 & $x^{6}+x^{4}+x^{2}-2 x+1$ \\
\hline-141296 & $-2^{4} \times 8831$ & S6 & 1 & $x^{6}+x^{5}+5 x^{4}+4 x^{3}+3 x^{2}+3 x+1$ \\
\hline-142343 & $-137 \times 1039$ & S6 & 1 & $x^{6}+3 x^{5}+5 x^{4}+7 x^{3}+8 x^{2}+5 x+2$ \\
\hline-142391 & -142391 & S6 & 1 & $x^{6}+2 x^{5}+2 x^{4}+x^{3}+3 x^{2}+4 x+3$ \\
\hline-142587 & $-3^{3} \times 5281$ & S6 & 1 & $x^{6}+x^{5}+2 x^{3}+x^{2}-3 x+1$ \\
\hline-142976 & $-2^{7} \times 1117$ & S6 & 1 & $x^{6}+2 x^{4}-2 x+1$ \\
\hline-143147 & $-43 \times 3329$ & 56 & 1 & $x^{6}+x^{5}+2 x^{2}-2 x+1$ \\
\hline-145903 & -145903 & S6 & 1 & $x^{6}+2 x^{5}+3 x^{4}+3 x^{3}+3 x^{2}+3 x+2$ \\
\hline-145927 & $-73 \times 1999$ & S6 & 1 & $x^{6}+x^{5}+x^{4}+3 x^{3}+6 x^{2}+4 x+1$ \\
\hline-145976 & $-2^{3} \times 71 \times 257$ & S6 & 1 & $x^{6}+2 x^{5}+2 x^{4}+3 x^{3}+3 x^{2}+2 x+2$ \\
\hline-146096 & $-2^{4} \times 23 \times 397$ & S6 & 1 & $x^{6}+x^{5}+6 x^{4}+3 x^{3}+10 x^{2}+x+5$ \\
\hline-146408 & $-2^{3} \times 18301$ & S6 & 1 & $x^{6}+x^{5}-3 x^{4}-x^{3}+7 x^{2}-6 x+2$ \\
\hline-147071 & $-61 \times 2411$ & s6 & 1 & $x^{6}+x^{5}+5 x^{4}+4 x^{3}+6 x^{2}+2 x+1$ \\
\hline-147136 & $-2^{6} \times 11^{2} \times 19$ & S6 & 1 & $x^{6}+2 x^{5}+2 x^{4}+2 x^{3}+5 x^{2}+4 x+1$ \\
\hline
\end{tabular}




\begin{tabular}{|c|c|c|c|c|}
\hline-147503 & -147503 & S6 & 1 & $x^{6}+x^{5}+3 x^{4}+5 x^{2}-x+2$ \\
\hline-149488 & $-2^{4} \times 9343$ & S6 & 1 & $x^{6}+3 x^{5}+7 x^{4}+10 x^{3}+12 x^{2}+8 x+2$ \\
\hline-149612 & $-2^{2} \times 113 \times 331$ & S6 & 1 & $x^{6}-x^{3}+2 x^{2}-x+1$ \\
\hline-150695 & $-5 \times 30139$ & s6 & 1 & $x^{6}+2 x^{5}+2 x^{4}+3 x^{3}+x^{2}-x+1$ \\
\hline-151087 & $-23 \times 6569$ & S6 & 1 & $x^{6}+x^{5}+5 x^{4}+5 x^{3}+6 x^{2}+4 x+1$ \\
\hline-151448 & $-2^{3} \times 11 \times 1721$ & S6 & 1 & $x^{6}+x^{5}+2 x^{3}+2 x^{2}-x+1$ \\
\hline-152704 & $-2^{7} \times 1193$ & S6 & 1 & $x^{6}+2 x^{5}+3 x^{4}+2 x^{3}+2 x^{2}+2 x+2$ \\
\hline-152728 & $-2^{3} \times 17 \times 1123$ & S6 & 1 & $x^{6}+3 x^{5}+7 x^{4}+10 x^{3}+12 x^{2}+9 x+4$ \\
\hline-153100 & $-2^{2} \times 5^{2} \times 1531$ & S6 & 1 & $x^{6}+2 x^{4}+3 x^{3}+4 x^{2}+3 x+1$ \\
\hline-153136 & $-2^{4} \times 17 \times 563$ & $S 6$ & 1 & $x^{6}+x^{5}+2 x^{4}-x^{3}+2 x^{2}-x+1$ \\
\hline-153331 & $-107 \times 1433$ & s6 & 1 & $x^{6}+2 x^{5}+x^{4}-x+1$ \\
\hline-155596 & $-2^{2} \times 7 \times 5557$ & S6 & 1 & $x^{6}+x^{5}+6 x^{4}+5 x^{3}+12 x^{2}+6 x+7$ \\
\hline-156919 & $-7 \times 29 \times 773$ & S6 & 1 & $x^{6}+5 x^{4}+x^{3}+10 x^{2}+2 x+7$ \\
\hline-157707 & $-3^{5} \times 11 \times 59$ & $\mathrm{~S} 6$ & 1 & $x^{6}+x^{5}-4 x^{4}-5 x^{3}+3 x^{2}+6 x+3$ \\
\hline-157847 & $-29 \times 5443$ & $\mathrm{~S} 6$ & 1 & $x^{6}+2 x^{5}+2 x^{4}-5 x^{3}-3 x^{2}+5 x+3$ \\
\hline-158083 & $-17^{2} \times 547$ & 56 & 1 & $x^{6}+2 x^{5}+3 x^{4}+5 x^{3}+6 x^{2}+3 x+1$ \\
\hline-158308 & $-2^{2} \times 19 \times 2083$ & $\mathrm{~S} 6$ & 1 & $x^{6}+2 x^{5}+2 x^{4}+3 x^{3}+x^{2}+2$ \\
\hline-159028 & $-2^{2} \times 83 \times 479$ & S6 & 1 & $x^{6}+x^{5}+4 x^{4}+2 x^{3}+5 x^{2}+2 x+2$ \\
\hline-160320 & $-2^{6} \times 3 \times 5 \times 167$ & S6 & 1 & $x^{6}+2 x^{5}+2 x^{4}+x^{2}+1$ \\
\hline-161484 & $-2^{2} \times 3 \times 13457$ & $\mathrm{~S} 6$ & 1 & $x^{6}+2 x^{5}+2 x^{4}+3 x^{3}-3 x+1$ \\
\hline-161659 & -161659 & S6 & 1 & $x^{6}+5 x^{4}+2 x^{3}+5 x^{2}+x+1$ \\
\hline-162299 & $-17 \times 9547$ & S6 & 1 & $x^{6}+2 x^{5}+6 x^{4}+5 x^{3}+8 x^{2}+2 x+3$ \\
\hline-162991 & $-389 \times 419$ & $\mathrm{~S} 6$ & 1 & $x^{6}+x^{5}+5 x^{4}+5 x^{3}+6 x^{2}+3 x+1$ \\
\hline-163635 & $-3 \times 5 \times 10909$ & S6 & 1 & $x^{6}+x^{5}+4 x^{4}+x^{3}+5 x^{2}+3$ \\
\hline-163915 & $-5 \times 32783$ & S6 & 1 & $x^{6}+x^{4}+2 x^{2}-3 x+1$ \\
\hline-165056 & $-2^{6} \times 2579$ & S6 & 1 & $x^{6}-4 x^{3}+8 x^{2}-6 x+2$ \\
\hline-165263 & $-7 \times 23609$ & S6 & 1 & $x^{6}+x^{5}+3 x^{4}+x^{3}+4 x^{2}+x+3$ \\
\hline-165611 & -165611 & S6 & 1 & $x^{6}+x^{5}+6 x^{4}+4 x^{3}+7 x^{2}+5 x+1$ \\
\hline-167231 & $-89 \times 1879$ & S6 & 1 & $x^{6}+x^{5}+5 x^{4}+9 x^{3}+8 x^{2}+4 x+1$ \\
\hline-167323 & $-13 \times 61 \times 211$ & S6 & 1 & $x^{6}+x^{5}+6 x^{4}+3 x^{3}+10 x^{2}+x+4$ \\
\hline-167351 & $-37 \times 4523$ & S6 & 1 & $x^{6}+x^{5}-5 x^{4}-6 x^{3}+5 x^{2}+9 x+4$ \\
\hline-168227 & -168227 & S6 & 1 & $x^{6}+x^{5}+2 x^{4}-x^{2}-x+1$ \\
\hline-168251 & $-311 \times 541$ & S6 & 1 & $x^{6}+3 x^{5}+x^{4}-4 x^{3}-x^{2}+2 x+1$ \\
\hline-170116 & $-2^{2} \times 71 \times 599$ & S6 & 1 & $x^{6}+x^{5}+6 x^{4}+4 x^{3}+7 x^{2}+2 x+2$ \\
\hline-170287 & $-13 \times 13099$ & S6 & 1 & $x^{6}-3 x^{3}+5 x^{2}-3 x+1$ \\
\hline-170432 & $-2^{6} \times 2663$ & S6 & 1 & $x^{6}+2 x^{5}+2 x^{4}-2 x^{3}-3 x^{2}-2 x+3$ \\
\hline-171371 & $-409 \times 419$ & S6 & 1 & $x^{6}+2 x^{5}+2 x^{4}+2 x^{3}-x+1$ \\
\hline-172031 & -172031 & S6 & 1 & $x^{6}+3 x^{5}+7 x^{4}+9 x^{3}+10 x^{2}+7 x+3$ \\
\hline-172039 & $-7^{2} \times 35.11$ & s6 & 1 & $x^{6}+x^{5}+4 x^{4}-3 x^{3}-x^{2}-x+1$ \\
\hline-172611 & $-3^{4} \times 2131$ & S6 & 1 & $x^{6}+x^{4}-x^{3}+x+1$ \\
\hline 173248 & $-2^{6} \times 2707$ & S6 & 1 & $x^{6}-2 x^{3}+2 x+1$ \\
\hline
\end{tabular}




\begin{tabular}{|c|c|}
\hline-174555 & $-3^{4} \times 5 \times 431$ \\
\hline-175688 & $-2^{3} \times 21961$ \\
\hline-176108 & $-2^{2} \times 44027$ \\
\hline-176836 & $-2^{2} \times 11 \times 4019$ \\
\hline-177259 & $-17 \times 10427$ \\
\hline-177311 & $-281 \times 631$ \\
\hline-177643 & $-401 \times 443$ \\
\hline-177656 & $-2^{3} \times 53 \times 419$ \\
\hline-178367 & $-7 \times 83 \times 307$ \\
\hline-180784 & $-2^{4} \times 11299$ \\
\hline-180848 & $-2^{4} \times 89 \times 127$ \\
\hline-182771 & $-193 \times 947$ \\
\hline-183728 & $-2^{4} \times 11483$ \\
\hline-183751 & $-53 \times 3467$ \\
\hline-184099 & $-47 \times 3917$ \\
\hline-185728 & $-2^{7} \times 1451$ \\
\hline-185743 & $-89 \times 2087$ \\
\hline-186560 & $-2^{6} \times 5 \times 11 \times 53$ \\
\hline-186712 & $-2^{3} \times 23339$ \\
\hline-187731 & $-3^{3} \times 17 \times 409$ \\
\hline-189015 & $-3 \times 5 \times 12601$ \\
\hline-189128 & $-2^{3} \times 47 \times 503$ \\
\hline-190235 & $-5 \times 38047$ \\
\hline
\end{tabular}

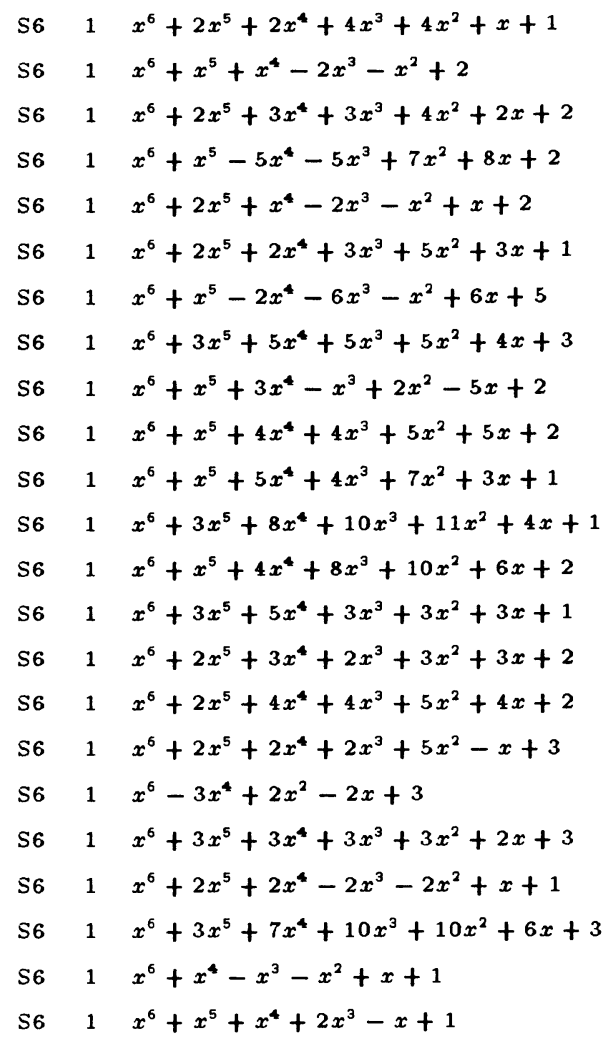

\section{CeReMaB}

C.N.R.S. U.A. 226

Université Bordeaux I

U.F.R. de Mathématiques et Informatique 351 cours de la Libération

33405 Talence FRANCE

E-mail: olivier@mizar.greco-prog.fr 